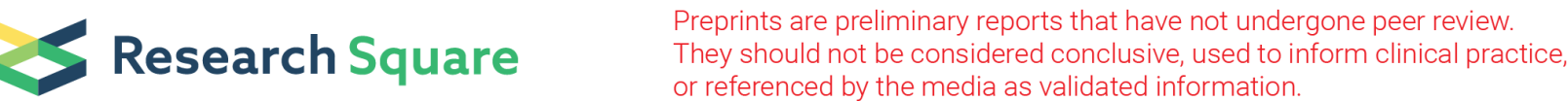

\section{Development and Validation of an Immune Microenvironment Based Prognostic Signature and Signal Pathways in Prostate Cancer}

Qi Zhao

Yangtze University Medical School

Yingying Cheng

Yangtze University Medical School

Yujie Ren

Yangtze University Medical School

Xianfa Li

Yangtze University Medical School

Ying Xiong ( $\nabla 1350266 @ q q . c o m)$

Yangtze University Medical School https://orcid.org/0000-0001-8615-4307

Primary research

Keywords: Prostate Cancer, TIICs, LTF, GM-CSF, JAK/STAT3, TCGA

Posted Date: November 11th, 2020

DOI: https://doi.org/10.21203/rs.3.rs-101879/v1

License: (c) (1) This work is licensed under a Creative Commons Attribution 4.0 International License.

Read Full License 


\section{Abstract}

\section{Background}

The study of the PCa immune microenvironment has brought new opportunities for the clinical diagnosis and treatment of PCa, but the current study has failed to obtain satisfactory results. We aim to develop a generalized, individualized immune prognostic signature that can improve the survival rate of patients.

\section{Methods}

We collected a total of 591 sample data from the TCGA and GEO cohorts, and evaluated the abundance and distribution of immune cell members in the mixed cell population in the gene expression matrix by CIBERSORT, ESTIMATE, sSGSEA, and other methods. Calculate the TMB value of the mutant gene to evaluate the reliability of $\mathrm{PCa}$ for immunotherapy. Besides, the genes related to the immune microenvironment and mutation load of PCa are obtained, and the target genes are obtained by the intersection with the immune-related genes in the ImmPort database. And perform pan-cancer analysis of the target gene to evaluate the immunological commonality and biological pathways in a variety of cancers. Finally, a Western blot experiment was used to explore the mechanism of the target gene in PCa immune microenvironment.

Results

We found that M0, M2, and Mast cell sresting differed significantly between prostate cancer and normal tissue and that patients with low plasma cell abundance were more likely to develop lymph node metastasis. LTF, as a core gene, is closely related to changes in the immune microenvironment of PCa, and its low expression in tumors promotes primary immune deficiency, cancer development, and enrichment in the JAK/STAT signaling pathway. Cell experiments found that LTF overexpression reduces tumor-derived GM-CSF secretion by inhibiting JAK/STAT pathway, achieving the purpose of regulating tumor immune microenvironment and inhibiting PCa cell proliferation and migration.

\section{Conclusions}

The proposed clinical-immune signature and core gene is the most promising biomarkers for improving the overall survival rate of PCa. It has been verified that overexpression of LTF reduces the secretion of tumor-derived GM-CSF by inhibiting the JAK/STAT pathway.

\section{Background}

Prostate cancer is a common male cancer in the world, and the advanced stage of prostate cancer has a poor prognosis, usually progressing to androgen resistant prostate cancer (CRPC) [1]. However, there is no radical treatment for CRPC at present, and the latest data show that the median overall survival time for CRPC is about 12.2 to 34.7 months [2]. 
Targeting the tumor-immune system is considered to be an influential direction in the development of anticancer drugs [3]. In recent years, several new types of immune system therapies, such as immune checkpoint blocking, cancer vaccines, and targeted antibodies, have shown significant effects on the treatment of tumors [4]. Sipuleucel-t, a personalized cancer vaccine targeting prostate acid phosphatase (PAP), became the first immunological agent approved by FDA to treat CRPC, thus, a large number of vaccine experiments targeting prostate-associated antigens became hot spots [5].In recent years, with the development of technology, bioinformatics has great advantages in $T$ cell receptor sequencing, immune microenvironment assessment [6], and neoantigens prediction, which brings new ideas for current immunotherapy.

At present, there is no biological pathway model based on bioinformatics to systematically evaluate the immune microenvironment of prostate cancer and study the genes related to the immune microenvironment of prostate cancer. Concerning the influence of TIIC infiltration on PCa and its relationship with patient prognosis and the exploration of genes related to the immune microenvironment, studies on one or two types of TIIC with only a small number of samples, or studies using only a single bioinformatics method without sufficient experimental verification may produce unreliable results. Therefore, systematic research is needed to explore relevant mechanisms by combining bioinformatics analysis and further validation based on cell experiments and external big data.

In this study, we collected data sets of prostate cancer expression spectrum and mutated genes in TCGA and GEO. CIBERSORT algorithm was used to determine the relative proportions of 22 kinds of TIIC(Tumorinfiltrating immune cells), explore the distribution of infiltrated immune cells in $\mathrm{PCa}$ and the correlation between TIIC and clinical factors, and Estimate algorithm was used to calculate PCa matrix and immune score. By extracting the genes related to PCa immune cell infiltration and mutation load, we excavated the genes most closely related to PCa immune microenvironment and analyzed the commonness of this gene in a variety of cancers. Besides, the biological pathway of target gene enrichment was analyzed based on GSEA, and the role of the target gene in the biological pathway of prostate cancer enrichment was explored by regulating the expression of the target gene in PCa cells and Western blot experiments. At the same time, the mechanism of the target gene in PCa immune microenvironment was studied. The flow chart of this study is shown in Figure $1(a, b, c, d, e)$.

\section{Materials And Methods}

\subsection{Data sources and cellular materials}

Gene expression profile data (GEPs data), gene mutation data, and clinical information of prostate cancer patients were obtained from TCGA (https://portal.gdc.cancer.gov/cart; as of May 1, 2020). Download RNA-Seq FPKM data of 499 PCa samples, 52 normal prostate samples, and SNV mutation data of 440 cases of prostate cancer. Also, these data sets contain clinical data of 551 patients, including sex, diagnostic age, T stage, survival status, and survival time in days. The GSE3325, GSE55945 data sets of prostate cancer in GEO are all based on the GPL570 platform, with a total of 40 samples, including 26 
tumor samples and 14 normal samples. Download the human genome annotation GTF file from the GenCode platform (http://www.gencodegenes.org/). We downloaded 2498 immune-related motifs through the immunology database and analysis website (ImmPort; https://www.immport.org/shared/genelists), which contains 17 immune categories based on various molecular functions $[7,8]$. The TSNAdb database (https://services.healthtech.dtu.dk/) contains somatic mutation data and human leukocyte antigen (HLA) allele information of 16 tumor types from 7748 tumor samples [9].

The main materials of cell experiment include PC-3 cells (prostate cancer cells $\triangle$ American Tissue Culture Collection) $₫$ Fetal bovine serum (Hyclone, Logan, UT, USA) $\triangle F 12 K$ medium, opti-MEM medium (GIBCO, Grand Island, USA) $₫$ Trypsin (Gibco, Grand Island, NY, USA)『Penicillin streptomycin (Sigma-Aldrich, St. Louis, MO॥XAnti-LTF antibody (ab109216), Anti-STAT3 antibody (ab119352), Anti-GM-CSF antibody (ab193345), Goat Anti-Mouse IgG H\&L (HRP) (ab205719), Goat Anti-Rabbit IgG H\&L (HRP) (ab205718), Goat Anti-Rat IgG H\&L (HRP) (ab205720) (Abcam, Cambridge, UK) هLipofectamine 2000 Transfection

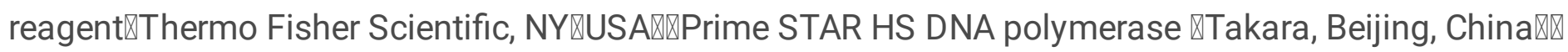

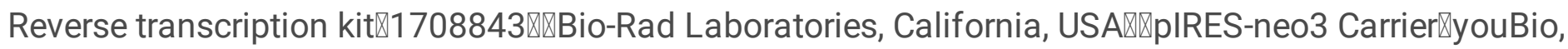
Changsha, China $\mathbb{\triangle}$ AFL II, Not I (New England Biolabs (Beijing) LTD, Beijing, China).

\subsection{Analysis of tumor immune cell infiltration based on CIBERSORT algorithm}

R software CIBERSORT package (version 3.6.3) was used to evaluate the relative expression level of 22 infiltrating immune fine cell types in PCa samples, namely [10]: naive B cells (Bn), Bm, plasma cells, CD8+T cells, naive CD4+T cells (CD4+Tn), CD4+ resting memory T cells (CD4+ Tmr), CD4+ memory activated T cells (CD4+Tma), Tfh, Tregs, $\mathrm{Y} \delta \mathrm{T}$, resting natural killer cells (NKr), activated natural killer cells (NKa), monocytes, M0 macrophages (M0), M1 macrophages (M1), M2 macrophages (M2), resting dendritic cells $(\mathrm{DCr}), \mathrm{DCa}$, resting mast cells $(\mathrm{Mr})$, activated mast cells $(\mathrm{Ma})$, eosinophils, and neutrophils. Through CIBERSORT deconvolution algorithm, the data of PCa GEPs matrix (including data from TCGA and GEO) are compared with 22 kinds of TIICs characteristic matrix data from CIBERSORT (https://cibersortx.stanford.edu/). The relative expression of $22 \mathrm{TIICs}$ in each sample was determined. Significant results $(P<0.05)$ were selected for subsequent analysis.

\subsection{Analysis of tumor microenvironment based on ssGSEA}

Normalized PCa GEPs data were compared with the gene set using "GSVA" (R package). ssGSEA (single sample GSEA, http://software.broadinstitute.org/gsea/msigdb/index.jsp) classifies gene sets with common biological functions, chromosomal localization, and physiological regulation [12]. ssGSEA is an implementation method that is mainly proposed for a single sample that cannot be used for GSEA. The principle is similar to GSEA. SSGSEA calculates the rank value of each gene according to the expression profile file and then performs subsequent statistical analysis. We analyzed 29 immune-associated gene sets that represented diverse immune cell types, functions, and pathways. We used the sSGSEA to quantify the activity or enrichment levels of immune cells, functions, or pathways in the cancer samples. The following 29 types of immune-associated gene sets were obtained[13]: Mast cells, Type II IFN 
Reponse, Macrophages, Neutrophils, Parainflammation, CCR, Treg, APC co-stimulation, APC co inhibition, T helper cells, T cell co-inhibition, Check-point, T cell co-stimulation, TIL, DCs, HLA, pDCs, aDCs, Type I IFN Reponse, NK cells, Th2 cells, B cells, Tfh, iDCs, MHC class I, Th1 cells, CD8+ T cells, Cytolytic activity, Inflammation promoting. The PCa GEPs data were compared with the gene set using R software GSVA package (version 3.6.3). The results were divided into three groups according to immune activity clustering. Clustering analysis was realized by the $\mathrm{R}$ software sparcl package (version 3.6.2). Finally, the immune scores of the three groups of clustered data sets were scored by ESTIMATE algorithm [14], which were divided into high, medium, and low immunization evaluation groups. To predict the correlation between low, medium, and high immune scores and tumor purity, HLA, PD-1, and survival rate in prostate cancer, and to speculate the factors affecting the progression of PCa. The tumor microenvironment score of TCGA cohort GEPs data was calculated by ESTIMATE algorithm, including stromal cell score and immune cell score. According to the median score level, the tumor microenvironment score was divided into high score group and low score group, and then the difference was analyzed using the R software limma package (version 3.8) Wilcox test method. The genes that meet the critical criteria of $P<0.05$ and I log-fold-change $\mid>1.0$ were screened.

\subsection{Analysis of tumor mutation load based on bioinformatics}

It is reported that highly mutated tumors are more likely to carry new antigens [16] that make them targets for activating immune cells, and a load of new antigens is related to the response to immunotherapy [17]. In this experiment, $440 \mathrm{PCa}$ samples (including 187748 mutant genes) were extracted from the TCGA database, and the R software maftools package (version 3.6.2) was used to visualize the data. The TMB value of each sample of prostate cancer was calculated by the Perl language (https://www.perl.org/) (version v5.30.0) [18]. The PCa GEPs data were divided into high TMB group and low TMB group according to $T M B$ value, and the relationship between $T M B$ value and lymphatic metastasis was analyzed. Rank sum test was used to analyze the difference of PCa GEPs between high and low TMB groups, and the genes that met the critical criteria of $P<0.05$ and $\mid \log$ fold-change $\mid>1.0$ were screened.

\subsection{Acquisition of key genes in prostate cancer}

First of all, the differential genes obtained by the microenvironment and TMB were analyzed by Venn analysis [19] (http://bioinformatics.psb.ugent.be/webtools/Venn/) to obtain the overlapping genes. Then, the immune-related genes and intersection genes were searched from the ImmPort database (https://www.immport.org/shared/genelists) and analyzed again by Venn, and the target genes related to PCa immune microenvironment were obtained. Finally, univariate logistic regression analysis was performed on the candidate genes based on the TNM staging data of prostate cancer respectively to evaluate the correlation between the screened genes and lymph node metastasis[20,21], and the highly correlated genes were most likely to be related to PCa progression, so it could be considered that this gene was the most likely target gene-related to immunity in the progression of prostate cancer.

\subsection{Verification and Analysis of LTF Gene based on pan-cancer}


Download 33 main tumor transcriptome data (FPKM), mutation data (varscan), and clinical data from the TCGA database on the UCSCXena website, and use Perl software to extract LTF expression matrix for the following analysis. The specific tumors are shown in Table 1.

The difference of LTF expression matrix between the normal group and tumor group was analyzed based on the Wilcox test, and the expression of LTF in each tumor was visualized by the R software ggpubr package (version 3.6.3).

The PFI survival status and survival time of each tumor in the clinical information of TCGA were extracted. Using R software survival, survminer, and forestplot package (version 3.6.3), the LTF expression matrix was combined with the PFI survival data of each sample. According to the median level of LTF expression, the samples were divided into high and low groups to obtain PFI survival curve. Finally, the effect of a gene on survival was described based on the Cox proportional hazards model, and a forest plot was drawn.

Perl software was used to calculate TMB values of mutation data from a total of 10114 samples of 33 tumors. Spearman packet (version 3.6.3) was used to test the correlation between LTF expression matrix and TMB values. Finally, FMSB packet (version 3.6.3) was used to visualize the TMB correlation of LTF in each tumor and draw the TMB correlation radar map. The TUMOR MSI data were extracted from TCGA, and a total of 10,416 MSI samples were collected. Then, the correlation test of LTF in each tumor MSI was performed with the above TMB correlation analysis method, and the results were visualized.

Microenvironment scores were calculated for 33 tumor samples and matched with LTF expression matrix. Finally, the correlation test of LTF expression matrix and microenvironment score was conducted based on The R language Spearman package (version 3.6.3), and the test threshold was $P<0.05$. The correlation of LTF in each tumor microenvironment score was visualized by ggPLOt2, GGPUBR, and ggExtra packages (version 3.6.3).

\subsection{LTF gene enrichment analysis (GSEA)}

To study the potential molecular mechanism of the key genes we screened, we carried out gene set enrichment analysis (https://www.gsea-msigdb.org/gsea/index.jsp) including KEGG analysis (C2), GO analysis (C5), and carcinogenic characteristic analysis of gene set (C6). Set the number of repeated permutations of GSEA software to 1000 , pvalue $<5 \%$, qvalue $<25 \%$ to filter the results of $\mathrm{C} 2, \mathrm{C} 5$ and $\mathrm{C} 6$. The GSEA analysis composite diagram is generated by R software packages "plyr", "ggplot2" and "grid".

\subsection{Cell culture}

PC-3 cells were subcultured in DMEM (GIBCO, Grand Island, USA) medium containing penicillin (final concentration $100 \mathrm{U} / \mathrm{mL}$ ), streptomycin (final concentration $100 \mu \mathrm{g} / \mathrm{mL}$ ), and 10\% FBS (Hyclone, Logan, UT, USA).

\subsection{Plasmid construction}




\subsubsection{Total RNA extraction and PCR amplification}

Total RNA was isolated from tumor tissues and cells with Trizol reagent (Invitrogen, USA). Reverse transcription was used to obtain cDNA, in $20 \mu \mathrm{I}$ system, and an appropriate amount of cDNA was used in the PCR experiment, and PCR amplification was performed in a 25ul system. All the primers used were by the principle of primer design. The primers were synthesized by Nanjing Jin si Rui Biotechnology Co., Ltd. (youBio, Changsha, China). The specific primers are shown in Table 2.

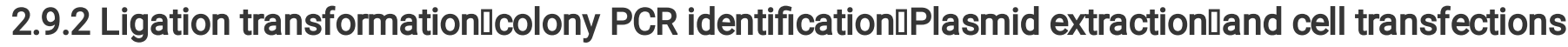

The vector linearized by double enzyme digestion and annealed double-stranded DNA were connected by T4DNAligase. The identified positive clones were inoculated in LB liquid medium containing Amp antibiotics, cultured at $37^{\circ} \mathrm{C}$ for 16 hours, and the appropriate amount of bacterial solution was sequenced. The plasmid was extracted from the bacterial solution with correct sequencing, and $0.8 \mu \mathrm{g}$ plasmid was dissolved in $50 \mu$ lopti-MEM serum-free medium to prepare reagent A. Reagent $B$ was prepared by dissolving $2 \mu$ I lipo2000 in $50 \mu$ I opti-MEM serum-free medium. The follow-up experiment was carried out 48 hours after transfection. The sequence of the carrier is shown in figure 2.

\subsubsection{Western blotting}

Protein samples were extracted using RIPA cell lysis buffer and protease inhibitor and phosphatase inhibitor (Beyotime Biotechnology, Shanghai, China). The total protein content ((Beyotime Biotechnology, China)) was determined by BCA method. The protein samples were electrophoretic on $10 \%$ sodium dodecyl sulfate-polyallylamide (SDS-PAGE) gel, transferred to polyvinylidene fluoride (PVDF) membrane, sealed with $5 \%$ skimmed milk, diluted with TBST to an appropriate concentration of primary antibody (LTF, STAT3, 1: 5000 GM-CSF, 1: 500), and incubated overnight at $4{ }^{\circ} \mathrm{C}$. Cut open the self-sealing bag and wash the film with TBST 3 times. Then the cells were incubated with an appropriate concentration of secondary antibody (1RV 5000) at room temperature for $1 \mathrm{~h}$. Finally, the luminous imaging station Tanon 6600 is used to detect the optical density, and the Image-Pro Plus software is used to analyze the optical density. According to the statistical difference between groups, one-way ANOVA and Tukey, s test was used, $\mathrm{P}<0.05$. It was considered that there was a significant difference.

\section{Results}

\subsection{Distribution of TIIC in prostate cancer}

In TCGA cohort, 44 tumor samples and 4 normal samples, and GEO cohort, 3 tumor samples and 7 normal samples met the screening criteria of CIBERSORT $P<0.05$. The proportion of macrophage $M 0$ and M 2 in TCGA cohort adenocarcinoma tissues was significantly higher than that in normal tissues, M2 macrophage in GEO cohort was also highly expressed in the tumor group, while Mast cells resting was

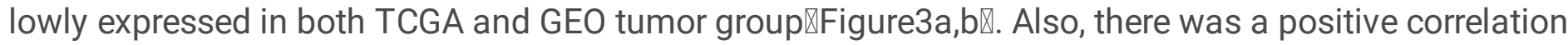
between plasma cell ratio and lymphatic metastasis (Figure4). 


\subsection{The immune microenvironment of prostate cancer}

According to the clustering of 29 immune-related gene sets, the immune activity scores of PCa samples were divided into low, medium, and high immunoactivity groups by ESTIMATE analysis. The results showed that the immune activity of the sample was positively correlated with the score of immune cells,

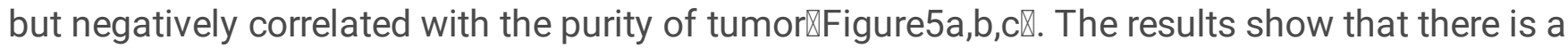
significant correlation between the progression of prostate cancer and immune microenvironment. Furthermore, the expression of immune-related genes (HLA, PD-L1 (CD274)) in prostate cancer was evaluated. It was found that all HLA genes were significantly different among the three groups, and the expression of the HLA gene increased with the enhancement of immune activity. Besides, the expression of the PD-L1 (CD274) gene increased with the enhancement of immune activity (Figure6a, b).

Based on the immune score and matrix score of PCa immune microenvironment, there were 963 upregulated genes, 2 down-regulated genes, and 91 up-regulated genes, and 3 down-regulated genes. Then 549 up-regulated genes and 1 down-regulated gene were obtained by Venn analysis $\triangle$ Figure7a,b区. The GO and KEGG analysis of these differential gene sets showed that the biological process of the differential gene set was involved in immune response, inflammatory response, and intercellular signal transduction. The main location areas are located in the external side of the plasma membrane $\mathbb{\text { immunological }}$

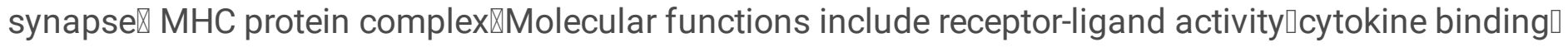
immunoglobulin binding $\square G$ protein-coupled nucleotide receptor activity. The main biological pathways involve cytokine-cytokine receptor interaction and cell adhesion molecule CAMS (Figure8a, b). According to the results of GO and KEGG, the above differential genes are closely related to the immune and inflammatory response in prostate cancer.

\subsection{PCa tumor mutation burden (TMB)}

A total of 187748 PCa mutant genes in TCGA were obtained. the results of R software visualization showed that Variant Classification was dominated by Missense Mutation and, Variant Type was mainly SNP. The gene mutation rate of 484 samples was $59.92 \%$, and the top 10 genes of mutation rate included: TP53, SPOP, TTN, KMT2D, FOXA1, KMT2C, MUC16, SYNE1, ATM, CSMD3. Among them, KMT2 family genes not only have a high mutation rate but also co-occur with many genes, including FAT3 and KMT2C, ZFHX3, and KMT2D, RYR1, and KMT2D $\otimes F i g u r e 9 a, b, c \bigotimes$. To explore the relationship between TMB value and PCa lymph node metastasis, it was found that the TMB value in the N1 stage was higher than that in the N0 stage in TNM staging $\triangle$ Figure10区. The results showed that there was a positive correlation between high TMB and lymphatic metastasis, which led to a poor prognosis of prostate cancer. Also, 74 down-regulated genes and 72 up-regulated genes were obtained by gene difference analysis between high and low TMB value groups of PCa expression profiles.

\subsection{Screening of genes related to immune microenvironment}

Perform the following steps to screen genes associated with the immune microenvironment in the progression of prostate cancer. The overlap of these genes was obtained by Venn analysis of 
550differential genes in tumor immune microenvironment and 146differential genes in TMB. Finally, 2498 immune-related genes were retrieved from the ImmPort database to screen out the two genes most related to the immune microenvironment, CSF3, and LTF (Figure11a, b).

\subsection{Key genes related to prognosis in PCa immune microenvironment}

The TNM staging clinical data of TCGA cohort were used for logical regression analysis of CSF3 and LTF. The results showed that CSF3 had no statistical significance in the logical regression analysis of TNM staging. The OR values of LTF in the logical regression analysis of N0/N1 and T2/T3 were 0.641 and 0.650 respectively, and the $P$ values were approximately less than 0.05 , which was statistically significant (Table 3). It is suggested that LTF is related to the progression of PCa and lymphatic metastasis, so LTF can be used as a key gene related to prognosis in the immune microenvironment of prostate cancer.

\subsection{Analysis of LTF in 33 tumors}

The differential analysis of LTF expression matrix in a total of 11057 cases of 33 tumors $(T=10327$, $\mathrm{N}=730$ ) showed that LTF was differentially expressed in 20 tumors, 15 of which were statistically significant. In most of the tumors, the expression level was higher in the normal group than in the tumor group (Figure12). This indicated that LTF expression was inhibited in most tumors and upregulated in normal tissues.

The expression of LTF gene in ACC and PRAD is related to the survival of progression-free interval (PFI). From the survival curve, it can be seen that with time, the PFI rate of patients with low expression of LFT is more obvious than that of patients with high expression of $P F I, A C C \varangle P=0.007 \rrbracket, P R A D \otimes P=0.040 \rrbracket$. It shows that the high expression of LTF makes the tumor in continuous remission to a certain extent, which means that the symptoms are improved and the effect of chemotherapy is good. According to the forest plot, there was a correlation between the expression of LTF in PRAD and the survival of PFI, and LTF was a low-risk gene $(H R<1)$ (Figure13a, b, c).

The expression of LTF in 33 kinds of tumors was correlated with TMB values of 18 kinds of tumors and was negatively correlated except LGG. LTF was negatively correlated with MSI of 11 kinds of tumors including PRAD (Figure14a, b).

There was a positive correlation between LTF expression and immune microenvironment scores in 29 tumors. The expression of LTF in ACC, BLCA, KICH, KIRC, KIRP, and PRAD of urinary system tumors had significant statistical significance with the immune score (Table 4). The above results confirm that there is a strong correlation between the expression of LTF and the immune microenvironment in tumors.

\subsection{Biological pathway of LTF enrichment}

GSEA enrichment showed that LTF gene set was enriched in 249 cases with low expression and 250 cases with high expression. According to KEGG analysis, LTF is mainly concentrated in aminoacyl TRNA biosynthesis, mismatch repair, ubiquitin-mediated proteolysis, nucleotide excision repair, and other 
pathways in normal tissues. LTF is mainly concentrated in primary immunodeficiency, cytokine - cytokine receptor interaction, JAK-STAT signaling pathway, cell adhesion molecule CAMS, pathway in cancer, prostate cancer occurrence, and other pathways. According to GO analysis, it is known that LTF gene is mainly related to the migration of immune cells, the production of immune factors, and the regulation of inflammatory response in tumors. According to oncogenic signatures(C6) analysis, RPS14, PTEN, MEL18, BMI1, and EGFR were up-regulated in the characteristic changes of major tumor markers, while MYC, ESC was down-regulated. Through GSEA analysis, it is found that LTF is a protective gene in PCa, and its down-regulation will inhibit the normal immune response in the body, thus making cancer cells escape the immune process, the detailed table information of enrichment analysis is shown in Table 5a,b; Table 6,7 .

\subsection{Distribution of new antigens on LTF}

TSNAdb is a database dedicated to new tumor-specific antigens. New tumor antigens can be quickly searched through the TSNAdb database, which is helpful to find new targets for immunotherapy [9]. Potential new antigens of R332S mutant HLA-C*05:01, HLA-C*03:04, HLA-A*02:01, and D244E mutant

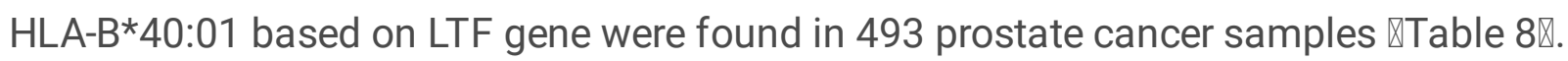

\subsection{Overexpression of LTF changes the state of the immune microenvironment in prostate cancer}

Our above study found that there is a close relationship between LTF gene and JAK/STAT signal pathway in the immune microenvironment of PCa. To further explore the mechanism, we overexpressed LTF gene in the prostate cancer PC-3 cell line and studied the effect of high expression of LTF on related proteins in JAK/STAT pathway by Western blot experiment. The results showed that compared with the pIRESneo3Control group, the content of LTF protein in the pIRESneo3-LTF group increased significantly, while the content of STAT3 and GM-CSF protein decreased significantly (Figure15). The results showed that after overexpression of LTF, GM-CSF secretion from the tumor was reduced through the JAK/STAT pathway, which could regulate the tumor immune microenvironment and inhibit tumor cell proliferation and migration.

\section{Discussion}

Some studies have suggested that PCa is an immunogenic disease and can be used as a model for studying targeted immunotherapy for therapeutic cancer $(2,22,23)$. Immune cell infiltration in tumor immune microenvironment is considered to play an important role in the biological behavior of many cancers $(14,24)$.

In this study, we found through bioinformatics methods that high immune score in the immune microenvironment was significantly correlated with poor prognosis in PCa patients, and the proportion of infiltrating macrophages $\mathrm{M} 0$ and $\mathrm{M} 2$ in PCa was significantly higher than that in normal tissues. This has aroused our interest in exploring the potential relationship between immune microenvironment and survival in patients with PCa. Some studies have shown that macrophages are an important part of the 
tumor microenvironment. M2 macrophages can alleviate inflammation and adaptive Th2 immunity and promote angiogenesis. In cancer, the tumor microenvironment makes the polarization balance of macrophages tend to M2 phenotype [25]. Besides, it has been confirmed that Dihydrotestosterone (DHT) increases the cytotoxic activity of macrophages through the up-regulation of TNF-related apoptosisinducing ligand (TRAIL), while castration induces the proliferation of androgen-resistant CaP cells by reducing the activity of macrophages $[26,27]$. This is consistent with our results that the proportion of macrophages $\mathrm{M} 0$ and $\mathrm{M} 2$ in $\mathrm{PCa}$ is significantly higher than that in normal tissues, androgen can activate macrophages, and the current observations may be of great significance for the implementation of immunotherapy in CaP.

Our study found that with the increase of immune activity of PCa tissue, the expression level of HLA and the expression of PD-L1 (CD274) gene also increased. However, Carretero et al observed that there was at least one change of HLA in $88 \%$ PCa tissue and the complete loss of HLA-1 expression in 50\%PCa tissue, which was related to tumor recurrence and high risk of D'Amico. Some studies have shown that tumorassociated macrophages express high levels of PD-1 [28] during tumor progression, and such macrophages usually show M2 phenotype, while the expression of PD-1-negative tumor-associated macrophages is similar to M1 macrophages [29]. It is suggested that the high expression of HLA can inhibit the cancer cells of PCa, and the positive expression of PD-1 will promote the chemotaxis of PCa infiltrating macrophages to the M2 phenotype.

In this study, we found that the proportion of Mast cells resting in PCa was lower than that in normal tissues. Also, there was a positive correlation between plasma cell level and tumor lymphatic metastasis. Some studies have found that in the early stage of tumor development, mast cells inhibit tumor growth by chemotactic CD8+T cells; with the further development of tumor, a large number of endogenous DAMP molecules in the tumor microenvironment change the characteristics of mast cells, chemotactic Treg cells infiltrate into the tumor area, thus promoting tumor growth [30]. The study of plasma cells in human HCC tissue by Wei et al showed that the increase of plasma cell level was related to the poor prognosis of patients [31]. It was confirmed that the change of Mast cells resting characteristics and the infiltration of a large number of plasma cells promoted the growth of PCa in PCa. Therefore, our study can guide the clinical practice of PCa immune targeted therapy and individualized therapy for patients with obvious immune cell infiltration.

High tumor mutation load (TMB) is an effective indicator of a new biomarker sensitive to immune checkpoint inhibitors [32]. In our study, we found several genes with the highest mutation rate of PCa TMB, such as TP53, SPOP, KMT2, and so on. Through co-expression analysis, we found that there was a co-expression between KMT2 family genes and many genes, among which FAT3 and KMT2C, ZFHX3, and KMT2D, RYR1 and KMT2D had obvious co-occurrence state. At the same time, we have found that ubiquitin-mediated proteolysis is up-regulated in PCa in GSEA analysis. According to literature reports, SPOP mutations promote the development of prostate cancer by changing the steady-state levels of key components in androgen signaling pathways. SPOP functional deletion mutations affect ubiquitinmediated PD-L1 degradation, resulting in an increase in PD-L1 levels in mouse tumors and primary 
human prostate cancer specimens and a decrease in the number of (TIL) in tumor-infiltrating lymphocytes $[33,34]$. It is also reported that KMT2C and KMT2D are the third and seventh most common mutations in cancer genes, which may lead to more cancer cell initialization and metastasis [35]. Therefore, further exploration of SPOP and KMT2 family mutant genes is helpful to understand the mechanism of PCa cell proliferation.

Through the pan-cancer analysis of a large number of data of 33 kinds of tumors, it was found that the expression of LTF gene was low in most tumors, including PCa, which confirmed that LTF was a tumor suppressor gene. It was found that there was a negative correlation between LTF gene and TMB and MSI in urinary tumors such as PCa $\triangle \mathrm{P}<0.001 \rrbracket$. The lower the expression of LTF in PCa, the higher the level of MSI. Microsatellite unstable (MSI) is an abnormal expression of the mismatch repair gene system in DNA replication and may be an important molecular event leading to the occurrence and development of tumors [36]. Some studies have shown that MSI has a high incidence in patients with low Gleason score or low serum PSA, which plays a certain role in the progression of tumors and is a sign of a good prognosis of prostate cancer [37]. PD-1 antibody pembrolizumab is approved for the treatment of solid tumors with microsatellite high instability (MSI-H) or mismatch repair defect (DMMR) [38,39]. In a retrospective study of $\mathrm{PCa}$, it was found that the effective rate of using the PD-1 antibody in patients with high microsatellite instability was more than $50 \%$ [40]. Using proactive tumor sequencing to screen the expression levels of MSIH/dMMR and LTF in all patients with advanced prostate cancer is of great significance for the personalized treatment of PCa patients [41,42]. Also, the PFI survival curve showed that over time, the PFI rate of patients with low expression of LFT in prostate cancer decreased more significantly than that of patients with high expression of PFI. Therefore, we speculate that the high expression of LTF can make the tumor remission to some extent. The above results provide effective support for us to explore the role of LTF in the progression of PCa. LTF can be used as an effective immune-related prognostic marker for PCa.

Based on GSEA analysis, we found that the high expression of LTF in tumors promoted aminoacyl TRNA biosynthesis, mismatch repair, ubiquitin-mediated proteolysis, nucleotide excision repair, and other pathways. The low expression of LTF is associated with primary immunodeficiency, cytokine - cytokine receptor interaction, JAK-STAT signaling pathway, cell adhesion molecule CAMS, cancer pathway, prostate cancer occurrence, and other pathways. It can be concluded that LTF is a protective gene in PCa, and its down-regulation will inhibit the normal immune response in the body, thus making cancer cells escape the immune process. More importantly, our study found that there is a close relationship between LTF gene and JAK/STAT signal pathway in the immune microenvironment of PCa. It has been reported in the literature that STAT3 phosphorylation is positively correlated with reduced survival, and activation of the STAT3 pathway promotes the development of androgen resistant prostate cancer (CRPC) [43]. Also, it has been found in animal experiments that blocking the activation of STAT3 can reduce inflammatory factors including GM-CSF [44]. Besides, a chronic inflammatory environment can promote tumor cells or tumor stromal cells to produce inflammatory factors, such as GM-CSF, high GM-CSF levels will recruit a large amount of MDSCs, and MDSCs in tumor immune microenvironment plays an important role in tumor immunity [45]. Therefore, we verified the expression of LTF, STAT3, and GM-CSF in PCa by Western 
blotting after overexpression of LTF gene in cell experiments. The experimental results verify our above analysis results, that is, the overexpression of LTF in PCa can affect the expression of STAT3 through the JAK/STAT pathway, to reduce the secretion of tumor-derived GM-CSF, and then interfere with the local immunosuppressive function of MDSCs. Achieve the purpose of regulating tumor immune microenvironment and inhibiting tumor cell proliferation and migration.

\section{Conclusion}

In summary, we analyzed the state of immune cell infiltration associated with PCa immune microenvironment and found that these infiltrating immune cells play a very important role in the progress of PCa, which provides a theoretical basis for the study of the mechanism of PCa immune microenvironment. Besides, we found that LTF gene is an important core gene in PCa immune microenvironment, and its low expression is closely related to poor prognosis. The pan-cancer analysis confirmed that the low expression of LTF gene in tumors promotes primary immunodeficiency, cancer development, and is closely related to JAK/STAT signaling pathway. More importantly, to further explore the effects of LTF gene and JAK/STAT signal pathway on the immune microenvironment of PCa, we verified that the overexpression of LTF in PCa suppressed the expression of STAT3 through the JAK/STAT pathway and reduced the secretion of tumor-derived GM-CSF, to regulate the tumor immune microenvironment and inhibit the proliferation and migration of tumor cells.

\section{Abbreviations}

PCa, Prostate Cancer; TCGA, The Cancer Genome Atlas; GEO, Gene Expression Omnibus; TMB, Tumor mutation burden; MSI, Microsatellite instability; GSEA, Gene set enrichment analysis; HLA, human leukocyte antigen; PD-L1, programmed death-ligand 1; PD-1, programmed cell death 1; CSF3, Colonystimulating factor 3; LTF, lactotransferrin; GM-CSF, Granulocyte-macrophage Colony Stimulating; CRPC, castrate-resistant prostate cancer; PAP, prostatic acid phosphatase; TIIC, Tumor-infiltrating immune cells; PFI, progression-free interval; SPOP,S peckle-type POZ protein; KMT2; Lysine methyltransferase, STAT3, signal transducer and activator of transcription 3; GEPs, gene expression profiles; Treg, regulatory $T$ cell

\section{Declarations}

\section{Acknowledgments}

We would like to thank the teachers of the Research Center of the First Affiliated Hospital of Yangtze University and Yangtze University School of Medicine for their help and the patients and researchers who participated in TCGA and GEO for providing the data.

\section{Authors' contributions}

QZ contributed to the study design. QZ, FL contributed to data collection. QZ, YYC, JR, and FL performed statistical analysis and interpretation. QZ drafted the manuscript. YYC, JR has verified the underlying 
data. All authors contributed to the critical revision of the final manuscript and approved the final version of the manuscript.

\section{Funding}

This study was supported by the Yangtze University School of Medicine and the First Affiliated Hospital of Yangtze University.

\section{Availability of data and material}

GEO datasets: https://www.ncbi.nlm.nih.gov/gds/.

TCGA dataset: https://portal.gdc.cancer.gov/.

ImmPort; https://www.immport.org/shared/genelists.

GenCode platform: http://www.gencodegenes.org/

The authenticity of this article has been validated by uploading the key raw data to the Research Data Deposit (RDD) public platform (www. researchdata. org.cn).

\section{Ethics approval and consent to participate}

The data used in our study were obtained from public databases TCGA and GEO, therefore, ethical approval was not required.

\section{Consent for publication}

The authors declare no conflicts of interest. All authors read and approved the final manuscript.

\section{Competing interests}

The authors have declared that they have no competing interest. The sources that funded this study played no role in the study design, data collection, data analysis, decision to publish, or preparation of the manuscript.

\section{Author details}

${ }^{1}$ School of Medicine, Yangtze University, No.1 Nanhu Rd, Jingzhou 434023, Hubei, China

${ }^{2}$ Department of Urology, The First Affiliated Hospital, School of Medicine, Yangtze University, No.6 Hangkong Rd, Jingzhou 434000, Hubei, China

\section{References}


1. Ji Z, Zhao W, Lin HK, Zhou X. Systematically understanding the immunity leading to CRPC progression. PLoS computational biology. 2019;15(9):e1007344.

2. Maia MC, Hansen AR. A comprehensive review of immunotherapies in prostate cancer. Critical reviews in oncology/hematology. 2017;113:292-303.

3. Hammerich L, Marron TU, Upadhyay R, Svensson-Arvelund J, Dhainaut M, Hussein S, et al. Systemic clinical tumor regressions and potentiation of PD1 blockade with in situ vaccination. Nature medicine. 2019;25(5):814-24.

4. Lee KL, Benz SC, Hicks KC, Nguyen A, Gameiro SR, Palena C, et al. Efficient Tumor Clearance and Diversified Immunity through Neoepitope Vaccines and Combinatorial Immunotherapy. Cancer immunology research. 2019;7(8):1359-70.

5. Noguchi M, Koga N, Moriya F, Itoh K. Immunotherapy in prostate cancer: challenges and opportunities. Immunotherapy. 2016;8(1):69-77.

6. Nishikawa H, Sakaguchi S. Regulatory T cells in cancer immunotherapy. Current opinion in immunology. 2014;27:1-7.

7. Chaussabel D, Baldwin N. Democratizing systems immunology with modular transcriptional repertoire analyses. Nature reviews Immunology. 2014;14(4):271-80.

8. Li S, Rouphael N, Duraisingham S, Romero-Steiner S, Presnell S, Davis C, et al. Molecular signatures of antibody responses derived from a systems biology study of five human vaccines. Nature immunology. 2014;15(2):195-204.

9. Wu J, Zhao W, Zhou B, Su Z, Gu X, Zhou Z, et al. TSNAdb: A Database for Tumor-specific Neoantigens from Immunogenomics Data Analysis. Genomics, proteomics \& bioinformatics. 2018;16(4):276-82.

10. Chen B, Khodadoust MS, Liu CL, Newman AM, Alizadeh AA. Profiling Tumor Infiltrating Immune Cells with CIBERSORT. Methods in molecular biology (Clifton, NJ). 2018;1711:243-59.

11. Newman AM, Liu CL, Green MR, Gentles AJ, Feng W, Xu Y, et al. Robust enumeration of cell subsets from tissue expression profiles. Nature methods. 2015;12(5):453-7.

12. Subramanian A, Tamayo P, Mootha VK, Mukherjee S, Ebert BL, Gillette MA, et al. Gene set enrichment analysis: a knowledge-based approach for interpreting genome-wide expression profiles.

Proceedings of the National Academy of Sciences of the United States of America. 2005;102(43):15545-50.

13. Ye L, Zhang T, Kang Z, Guo G, Sun Y, Lin K, et al. Tumor-Infiltrating Immune Cells Act as a Marker for Prognosis in Colorectal Cancer. Frontiers in immunology. 2019;10:2368.

14. Yoshihara K, Shahmoradgoli M, Martínez E, Vegesna R, Kim H, Torres-Garcia W, et al. Inferring tumour purity and stromal and immune cell admixture from expression data. Nature communications. 2013;4:2612.

15. Shah N, Wang P, Wongvipat J, Karthaus WR, Abida W, Armenia J, et al. Regulation of the glucocorticoid receptor via a BET-dependent enhancer drives antiandrogen resistance in prostate cancer. eLife. 2017;6. 
16. Chalmers ZR, Connelly CF, Fabrizio D, Gay L, Ali SM, Ennis R, et al. Analysis of 100,000 human cancer genomes reveals the landscape of tumor mutational burden. Genome medicine. 2017;9(1):34.

17. van Rooij N, van Buuren MM, Philips D, Velds A, Toebes M, Heemskerk B, et al. Tumor exome analysis reveals neoantigen-specific T-cell reactivity in an ipilimumab-responsive melanoma. Journal of clinical oncology : official journal of the American Society of Clinical Oncology. 2013;31(32):e439-42.

18. Endris V, Buchhalter I, Allgäuer M, Rempel E, Lier A, Volckmar AL, et al. Measurement of tumor mutational burden (TMB) in routine molecular diagnostics: in silico and real-life analysis of three larger gene panels. International journal of cancer. 2019;144(9):2303-12.

19. Bhattacharya S, Andorf S, Gomes L, Dunn P, Schaefer H, Pontius J, et al. ImmPort: disseminating data to the public for the future of immunology. Immunologic research. 2014;58(2-3):234-9.

20. Liu GM, Zeng HD, Zhang CY, Xu JW. Identification of a six-gene signature predicting overall survival for hepatocellular carcinoma. Cancer cell international. 2019;19:138.

21. Tibshirani R. The lasso method for variable selection in the Cox model. Statistics in medicine. 1997;16(4):385-95.

22. Baxevanis CN, Papamichail M, Perez SA. Prostate cancer vaccines: the long road to clinical application. Cancer immunology, immunotherapy : CII. 2015;64(4):401-8.

23. Comiskey MC, Dallos MC, Drake CG. Immunotherapy in Prostate Cancer: Teaching an Old Dog New Tricks. Current oncology reports. 2018;20(9):75.

24. Hanahan D, Coussens LM. Accessories to the crime: functions of cells recruited to the tumor microenvironment. Cancer cell. 2012;21(3):309-22.

25. Mantovani A, Locati M. Tumor-associated macrophages as a paradigm of macrophage plasticity, diversity, and polarization: lessons and open questions. Arteriosclerosis, thrombosis, and vascular biology. 2013;33(7):1478-83.

26. Lee GT, Kim JH, Kwon SJ, Stein MN, Hong JH, Nagaya N, et al. Dihydrotestosterone Increases Cytotoxic Activity of Macrophages on Prostate Cancer Cells via TRAIL. Endocrinology. 2019;160(9):2049-60.

27. Qian BZ, Pollard JW. Macrophage diversity enhances tumor progression and metastasis. Cell. 2010;141(1):39-51.

28. Li B, Song TN, Wang FR, Yin C, Li Z, Lin JP, et al. Tumor-derived exosomal HMGB1 promotes esophageal squamous cell carcinoma progression through inducing PD1(+) TAM expansion. Oncogenesis. 2019;8(3):17.

29. Gordon SR, Maute RL, Dulken BW, Hutter G, George BM, McCracken MN, et al. PD-1 expression by tumour-associated macrophages inhibits phagocytosis and tumour immunity. Nature. 2017;545(7655):495-9.

30. Song CW. The influence of tumor microenvironment on the role of mast cells in tumor growth [PhD]: Huazhong University of Science and Technology; 2010. 
31. Wei Y, Lao XM, Xiao X, Wang XY, Wu ZJ, Zeng QH, et al. Plasma Cell Polarization to the Immunoglobulin G Phenotype in Hepatocellular Carcinomas Involves Epigenetic Alterations and Promotes Hepatoma Progression in Mice. Gastroenterology. 2019;156(6):1890-904.e16.

32. Merino DM, McShane LM, Fabrizio D, Funari V, Chen SJ, White JR, et al. Establishing guidelines to harmonize tumor mutational burden (TMB): in silico assessment of variation in TMB quantification across diagnostic platforms: phase I of the Friends of Cancer Research TMB Harmonization Project. Journal for immunotherapy of cancer. 2020;8(1).

33. Mani RS. The emerging role of speckle-type POZ protein (SPOP) in cancer development. Drug discovery today. 2014;19(9):1498-502.

34. Zhang J, Bu X, Wang H, Zhu Y, Geng Y, Nihira NT, et al. Author Correction: Cyclin D-CDK4 kinase destabilizes PD-L1 via cullin 3-SPOP to control cancer immune surveillance. Nature. 2019;571(7766):E10.

35. Fagan RJ, Dingwall AK. COMPASS Ascending: Emerging clues regarding the roles of MLL3/KMT2C and MLL2/KMT2D proteins in cancer. Cancer letters. 2019;458:56-65.

36. Overman MJ, McDermott R, Leach JL, Lonardi S, Lenz HJ, Morse MA, et al. Nivolumab in patients with metastatic DNA mismatch repair-deficient or microsatellite instability-high colorectal cancer (CheckMate 142): an open-label, multicentre, phase 2 study. The Lancet Oncology. 2017;18(9):118291.

37. Abida W, Cheng ML, Armenia J, Middha S, Autio KA, Vargas HA, et al. Analysis of the Prevalence of Microsatellite Instability in Prostate Cancer and Response to Immune Checkpoint Blockade. JAMA oncology. 2019;5(4):471-8.

38. LaFleur MW, Muroyama Y, Drake CG, Sharpe AH. Inhibitors of the PD-1 Pathway in Tumor Therapy. Journal of immunology (Baltimore, Md : 1950). 2018;200(2):375-83.

39. Kantoff PW, Higano CS, Shore ND, Berger ER, Small EJ, Penson DF, et al. Sipuleucel-T immunotherapy for castration-resistant prostate cancer. The New England journal of medicine. 2010;363(5):411-22.

40. Sharma M, Yang Z, Miyamoto H. Immunohistochemistry of immune checkpoint markers PD-1 and PD-L1 in prostate cancer. Medicine. 2019;98(38):e17257.

41. Abida W, Armenia J, Gopalan A, Brennan R, Walsh M, Barron D, et al. Prospective Genomic Profiling of Prostate Cancer Across Disease States Reveals Germline and Somatic Alterations That May Affect Clinical Decision Making. JCO precision oncology. 2017;2017.

42. Zehir A, Benayed R, Shah RH, Syed A, Middha S, Kim HR, et al. Mutational landscape of metastatic cancer revealed from prospective clinical sequencing of 10,000 patients. Nature medicine. 2017;23(6):703-13.

43. Tam L, McGlynn LM, Traynor P, Mukherjee R, Bartlett JM, Edwards J. Expression levels of the JAK/STAT pathway in the transition from hormone-sensitive to hormone-refractory prostate cancer. British journal of cancer. 2007;97(3):378-83. 
44. Ganesan R, Rasool M. Interleukin 17 regulates SHP-2 and IL-17RA/STAT-3 dependent Cyr61, IL-23 and GM-CSF expression and RANKL mediated osteoclastogenesis by fibroblast-like synoviocytes in rheumatoid arthritis. Molecular immunology. 2017;91:134-44.

45. Zhao HC. CNOT7 gene knockdown reduces the effect of GM-CSF secretion on liver cancer tumor immune microenvironment through JAK/STAT pathway [Master]: Shanxi Medical University; 2019.

\section{Tables}

Due to technical limitations, table $1,2,3,4,5,6,7$ and 8 is only available as a download in the Supplemental Files section.

\section{Figures}

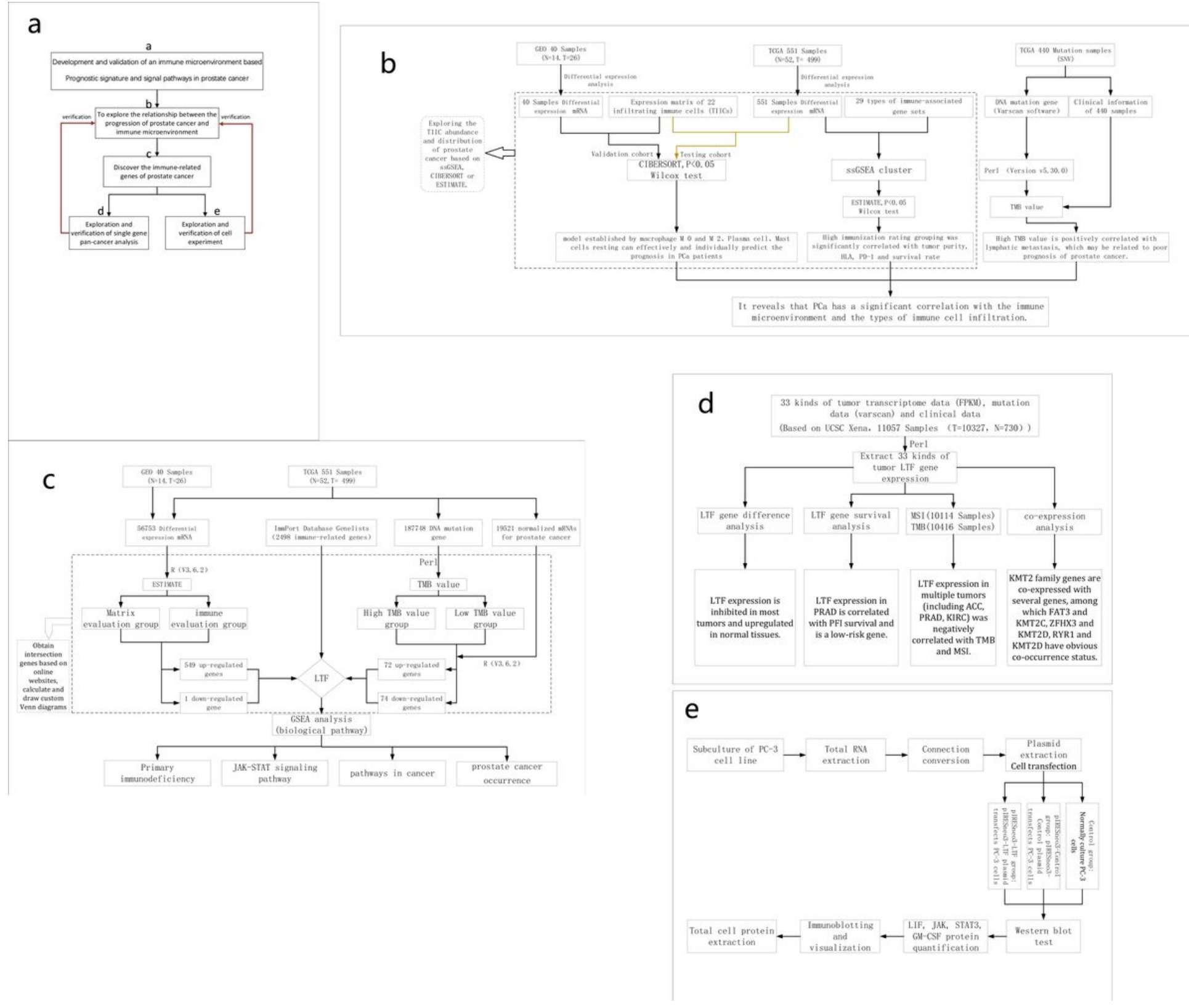


Figure 1

The flow chart of this study is shown in Figure $1(a, b, c, d, e)$.

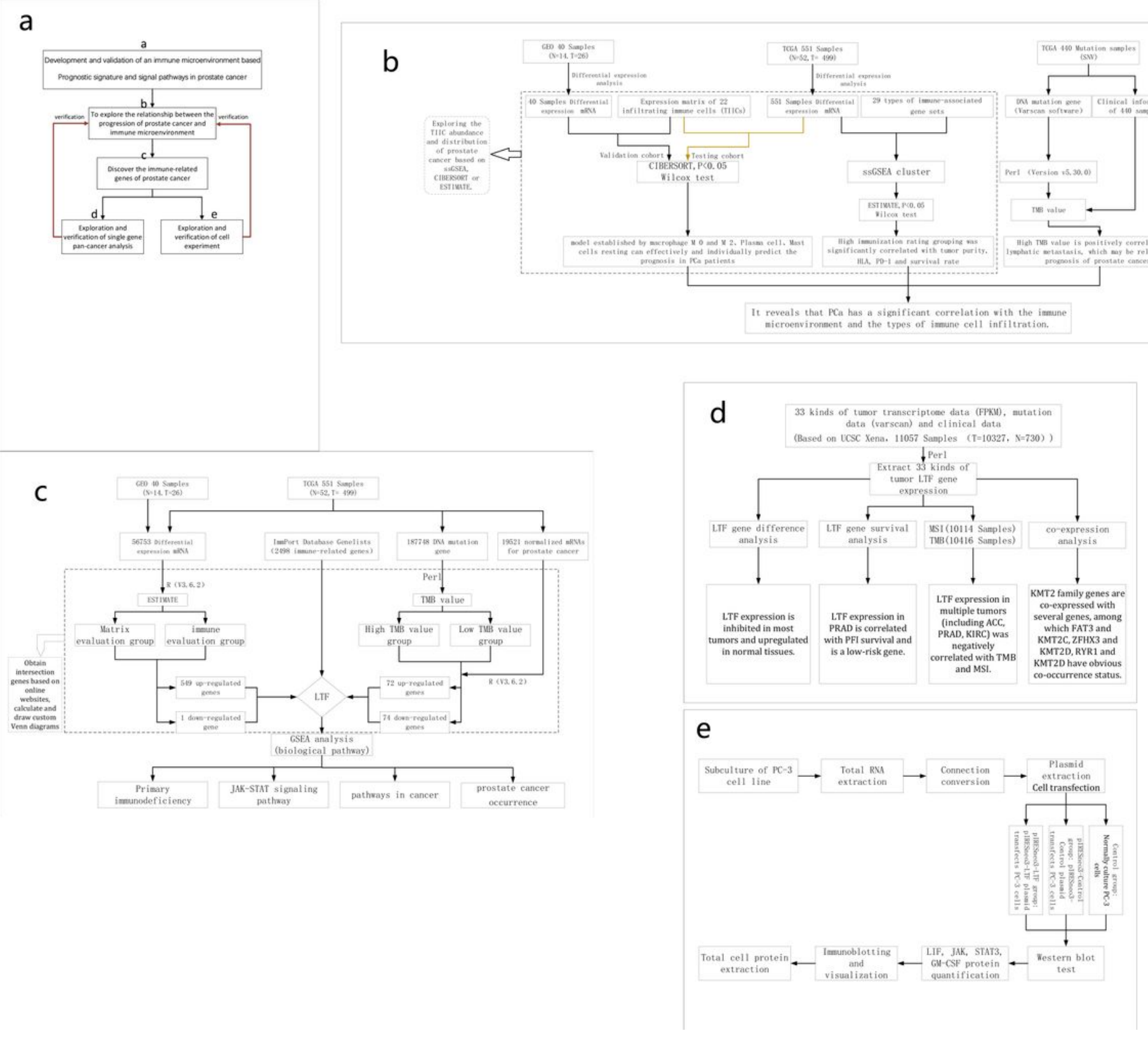

Figure 1

The flow chart of this study is shown in Figure $1(a, b, c, d, e)$. 


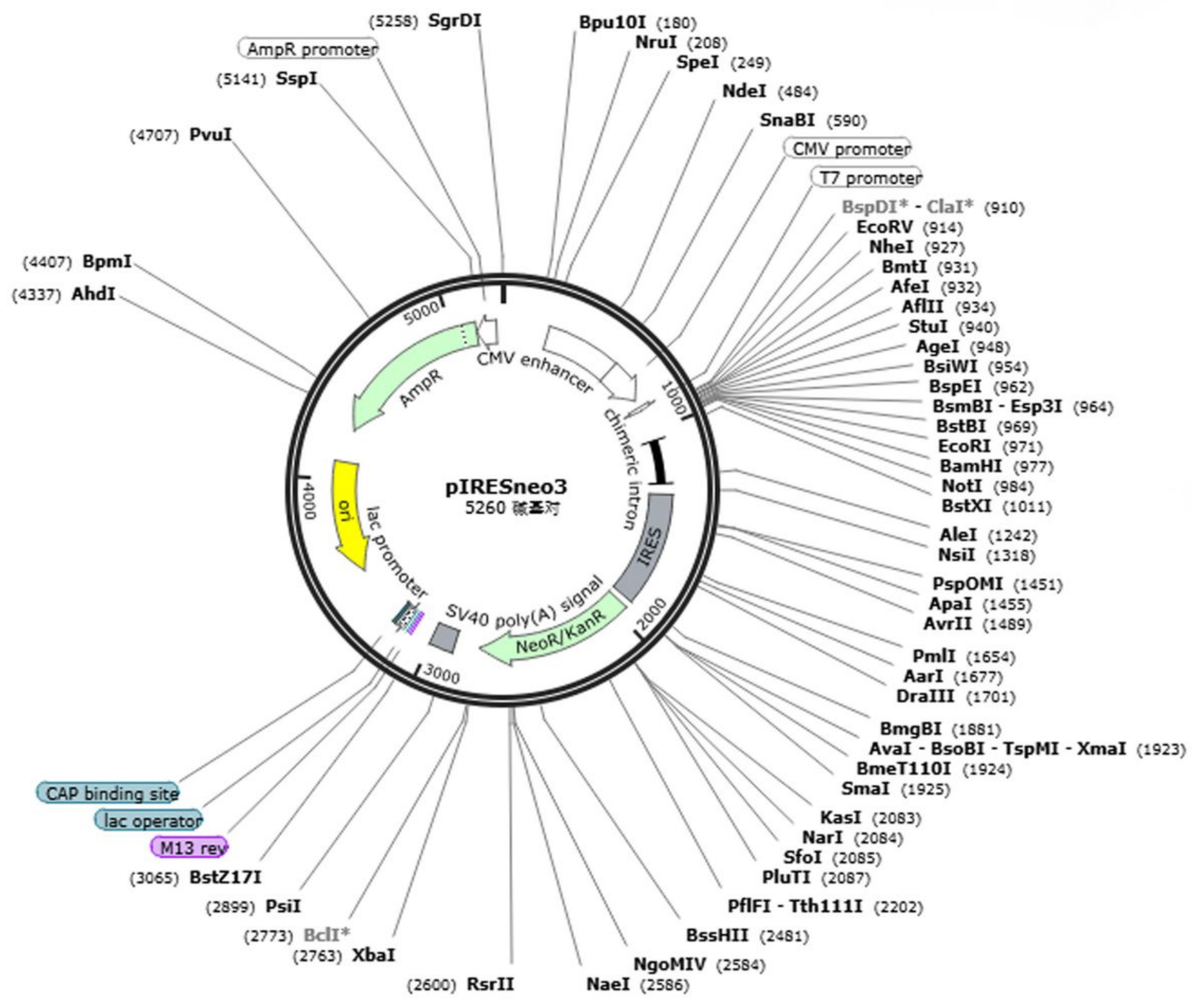

Figure 2

The follow-up experiment was carried out 48 hours after transfection. The sequence of the carrier is shown in figure 2. 


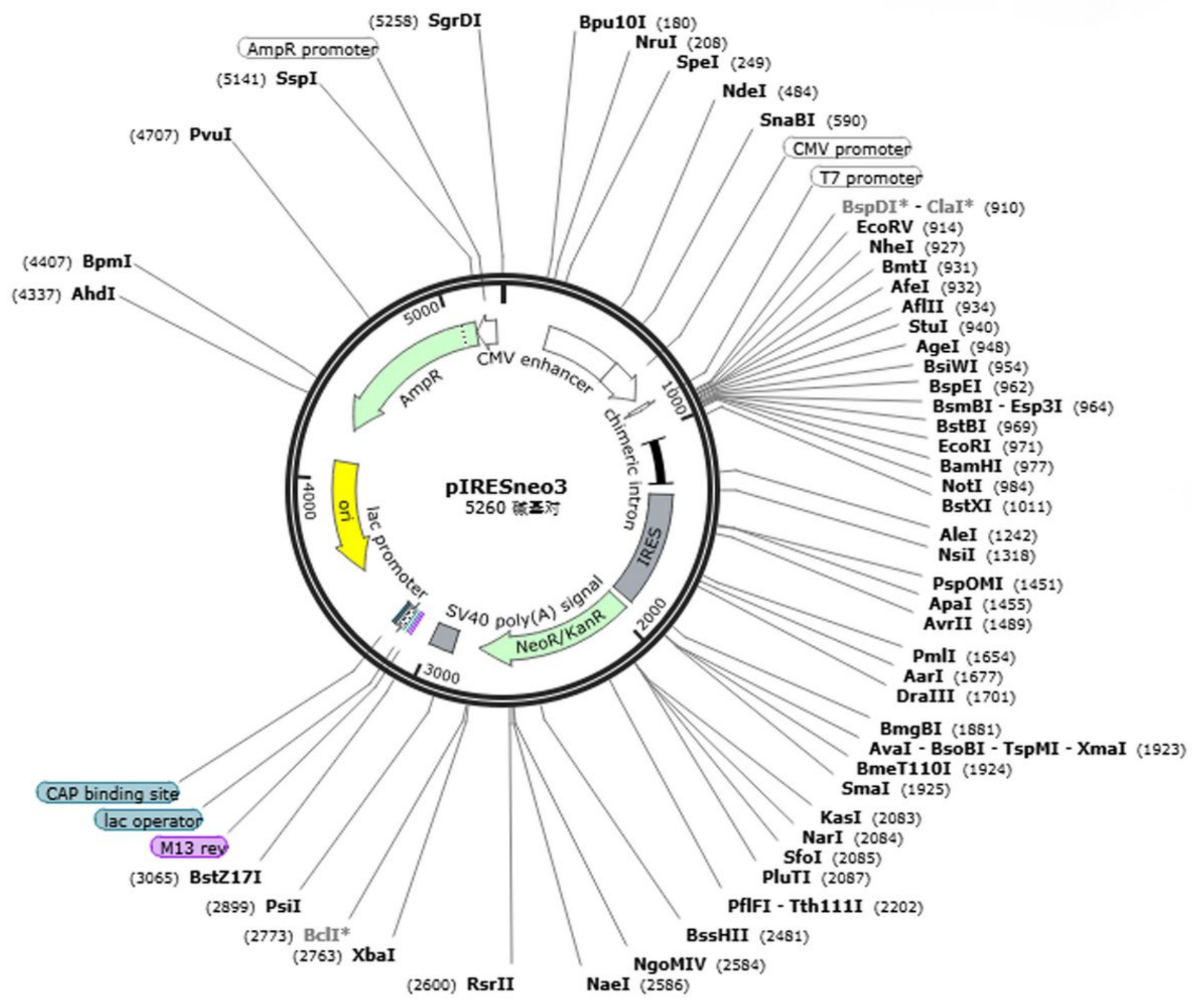

Figure 2

The follow-up experiment was carried out 48 hours after transfection. The sequence of the carrier is shown in figure 2. 

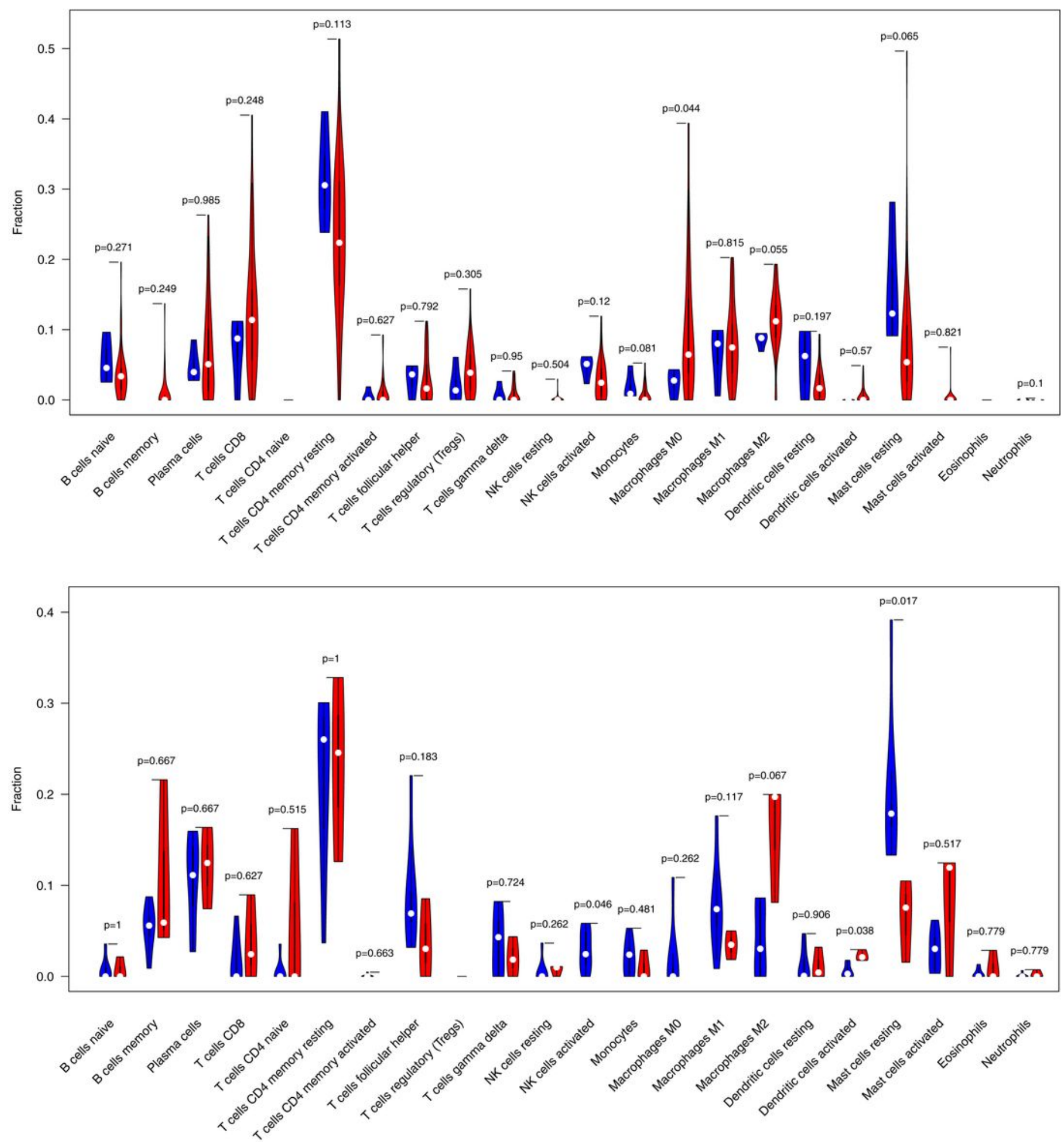

Figure 3

Mast cells resting was lowly expressed in both TCGA and GEO tumor group囚Figure3a,b 

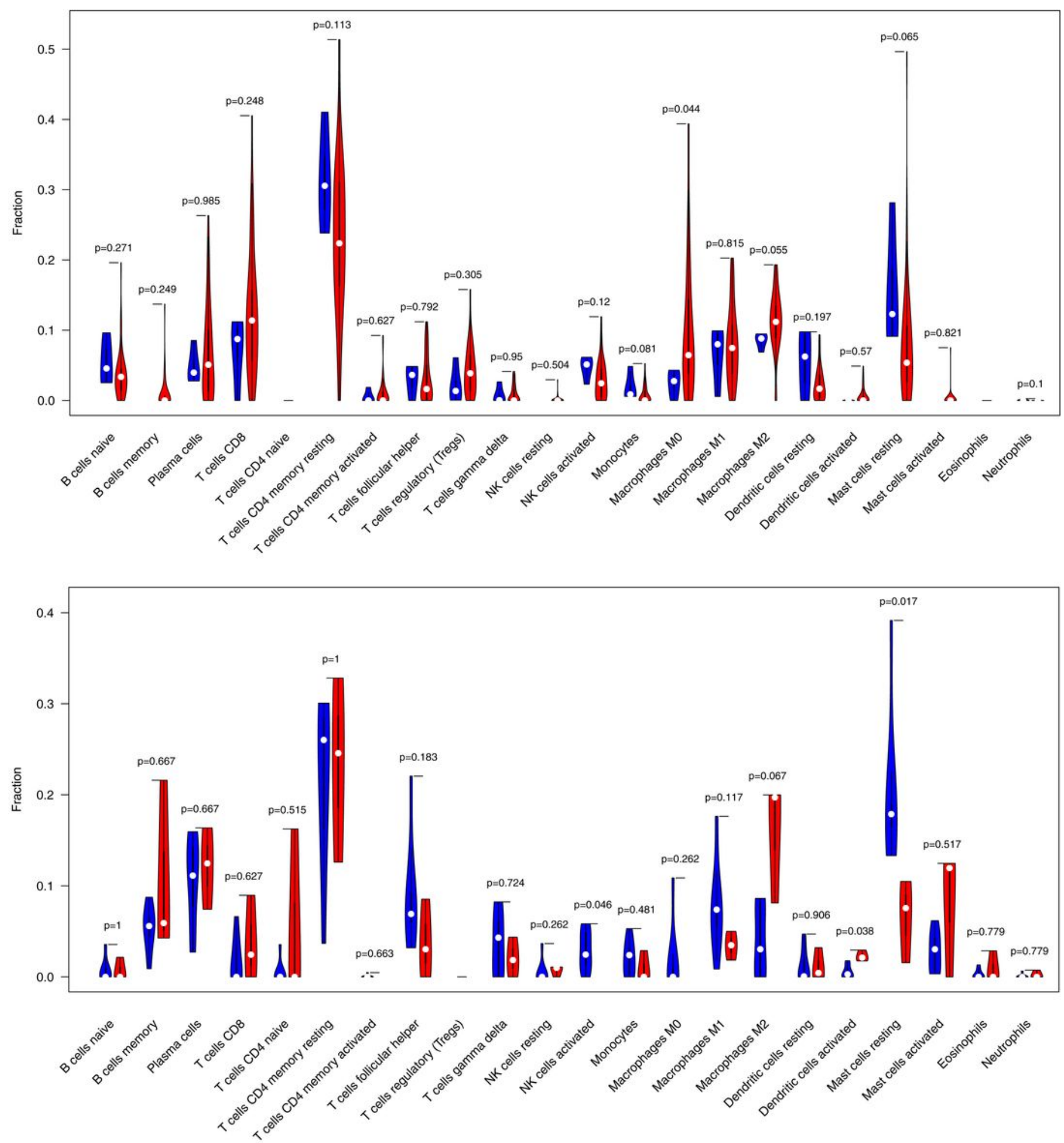

Figure 3

Mast cells resting was lowly expressed in both TCGA and GEO tumor group囚Figure3a,b 


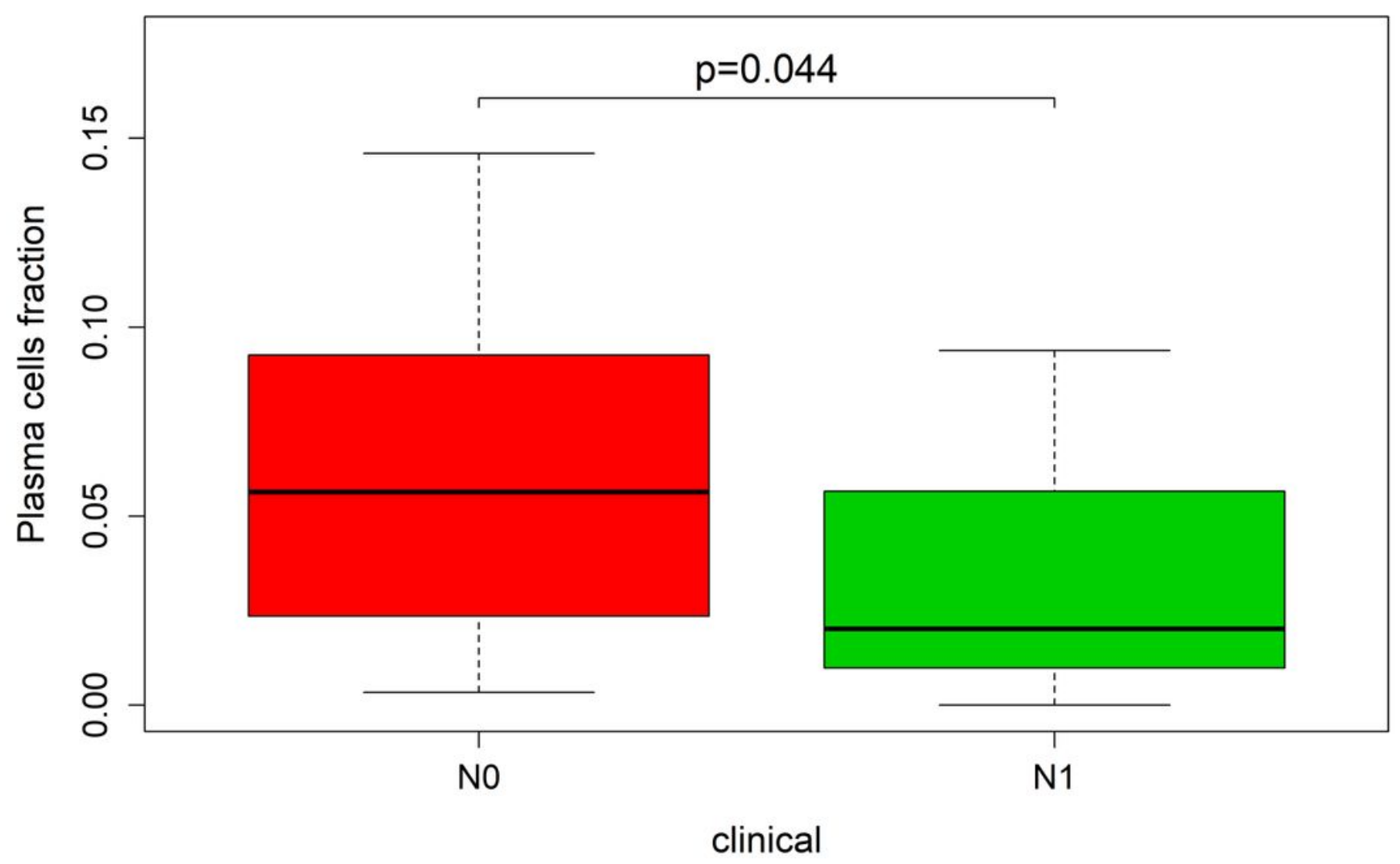

Figure 4

There was a positive correlation between plasma cell ratio and lymphatic metastasis (Figure4). 


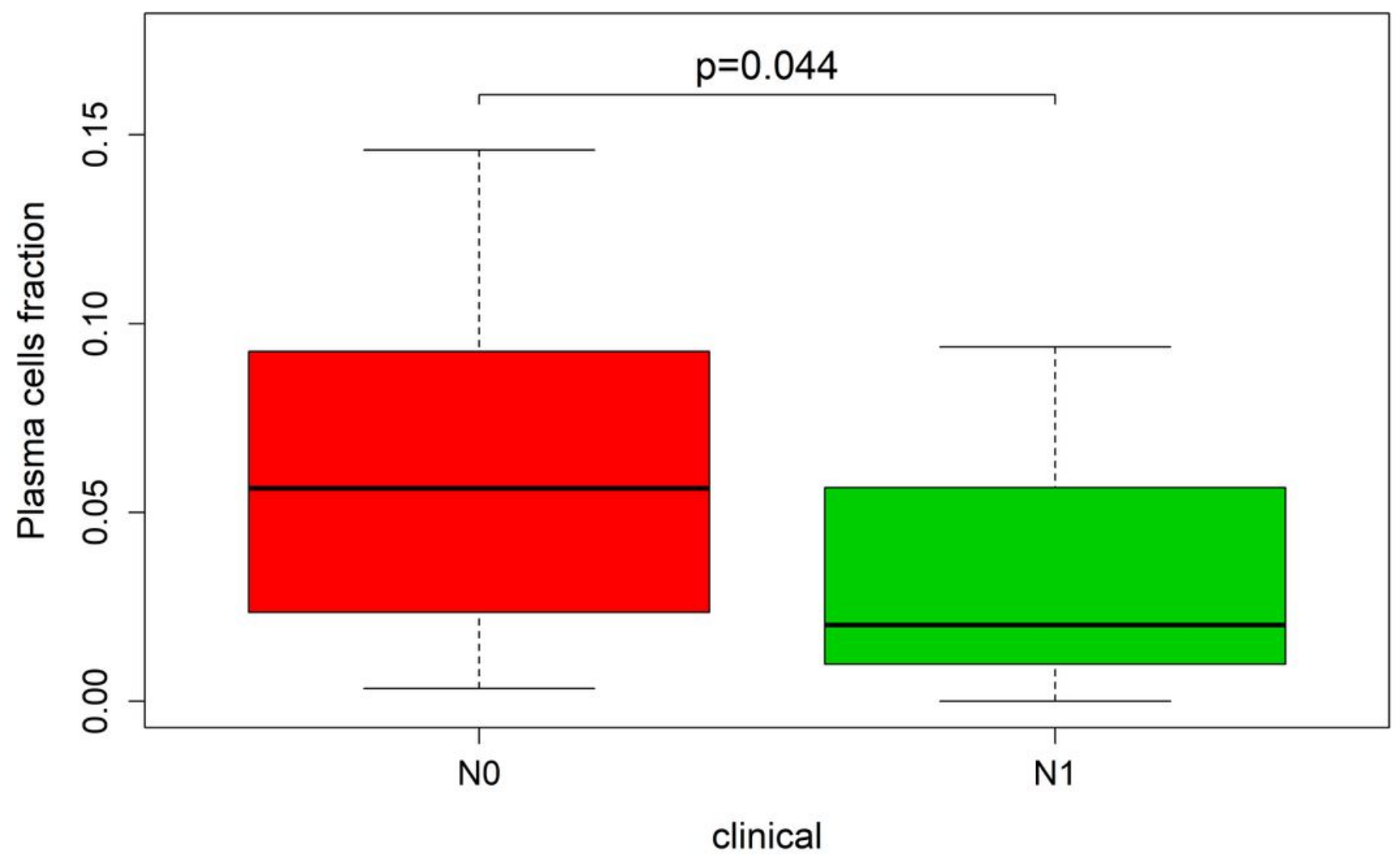

Figure 4

There was a positive correlation between plasma cell ratio and lymphatic metastasis (Figure4). 

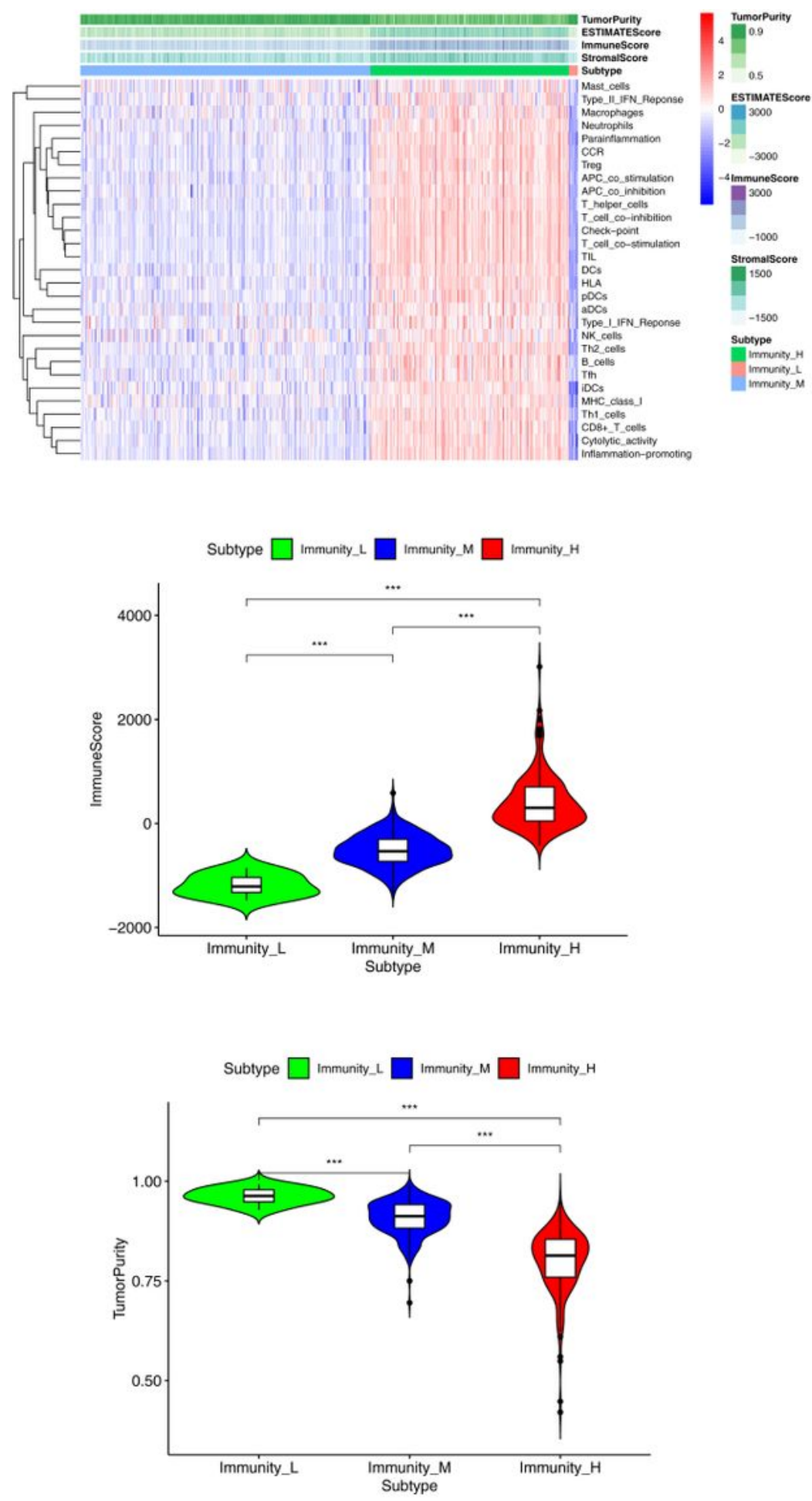

\section{Figure 5}

The results showed that the immune activity of the sample was positively correlated with the score of immune cells, but negatively correlated with the purity of tumorखFigure $5 a, b, c)$. 

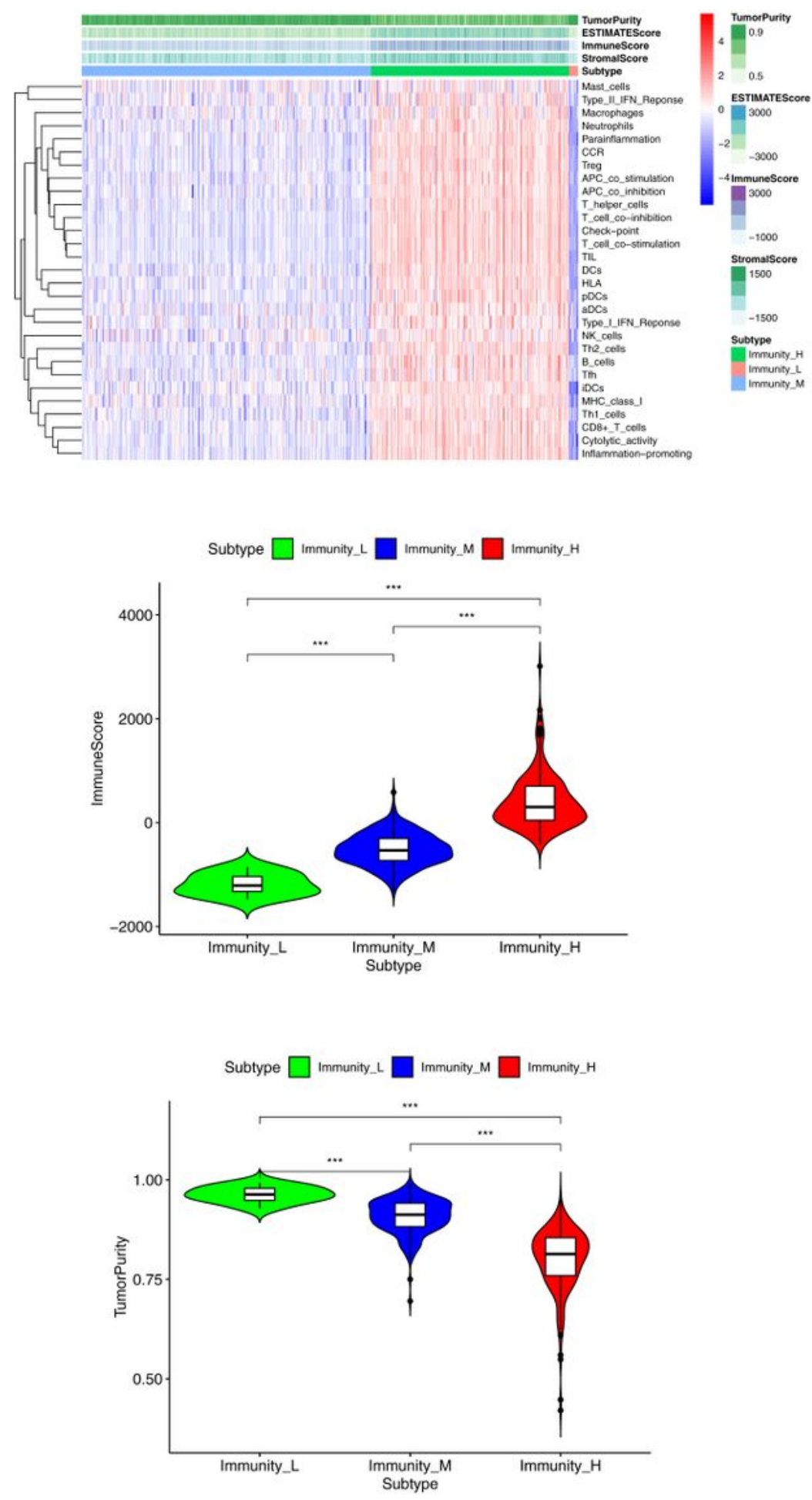

\section{Figure 5}

The results showed that the immune activity of the sample was positively correlated with the score of immune cells, but negatively correlated with the purity of tumor冈Figure5a,b,c). 


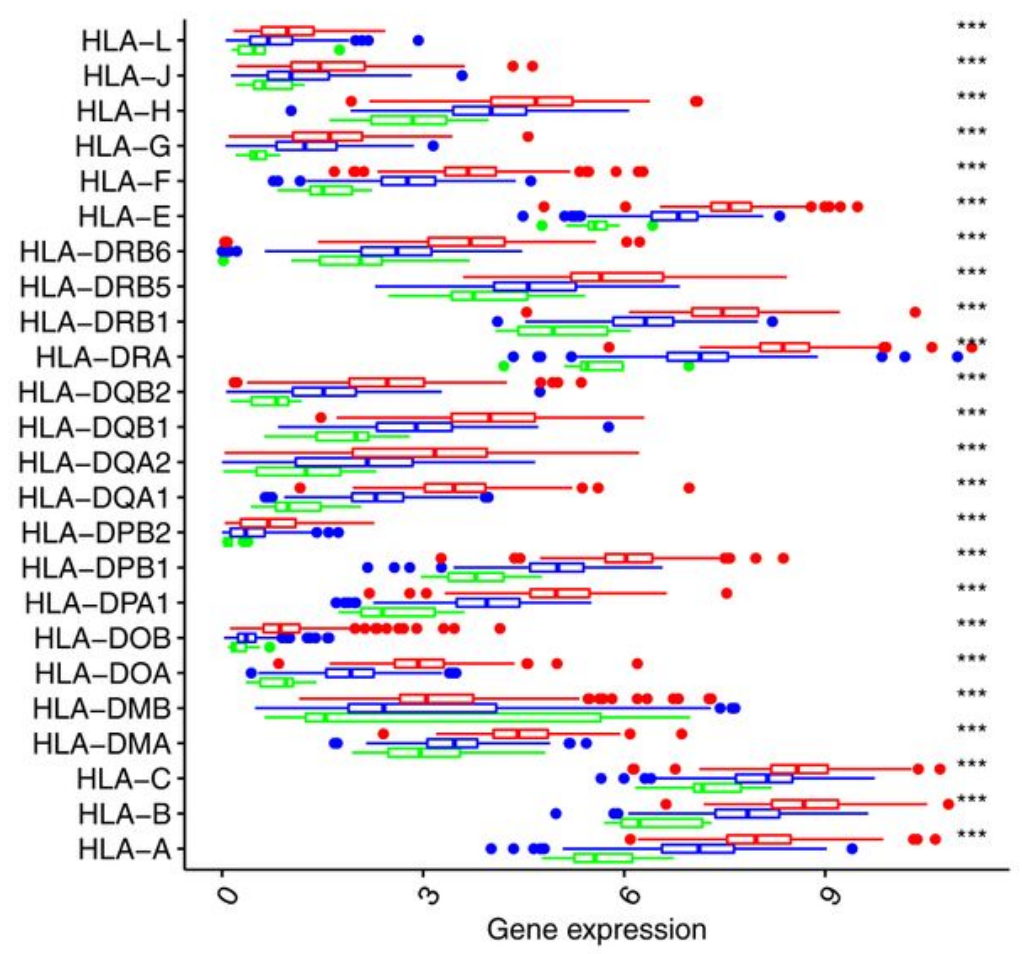

Subtype 自 Immunity_L Immunity_M 官 Immunity_H

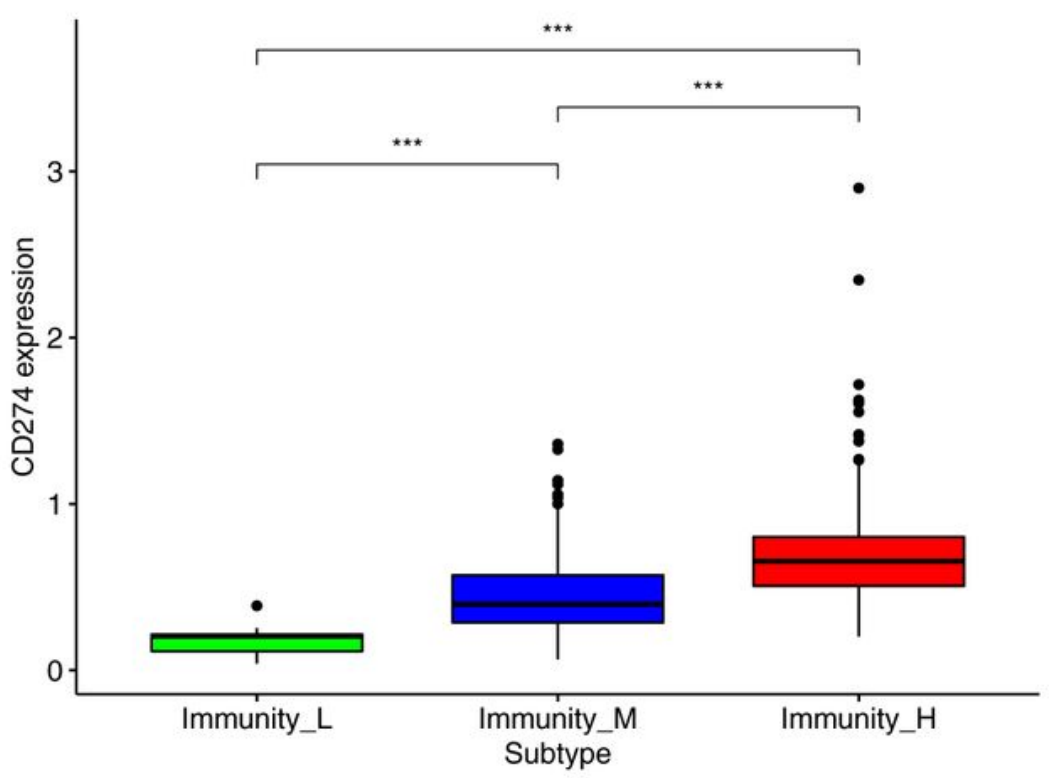

\section{Figure 6}

Besides, the expression of the PD-L1 (CD274) gene increased with the enhancement of immune activity (Figure6a, b). 


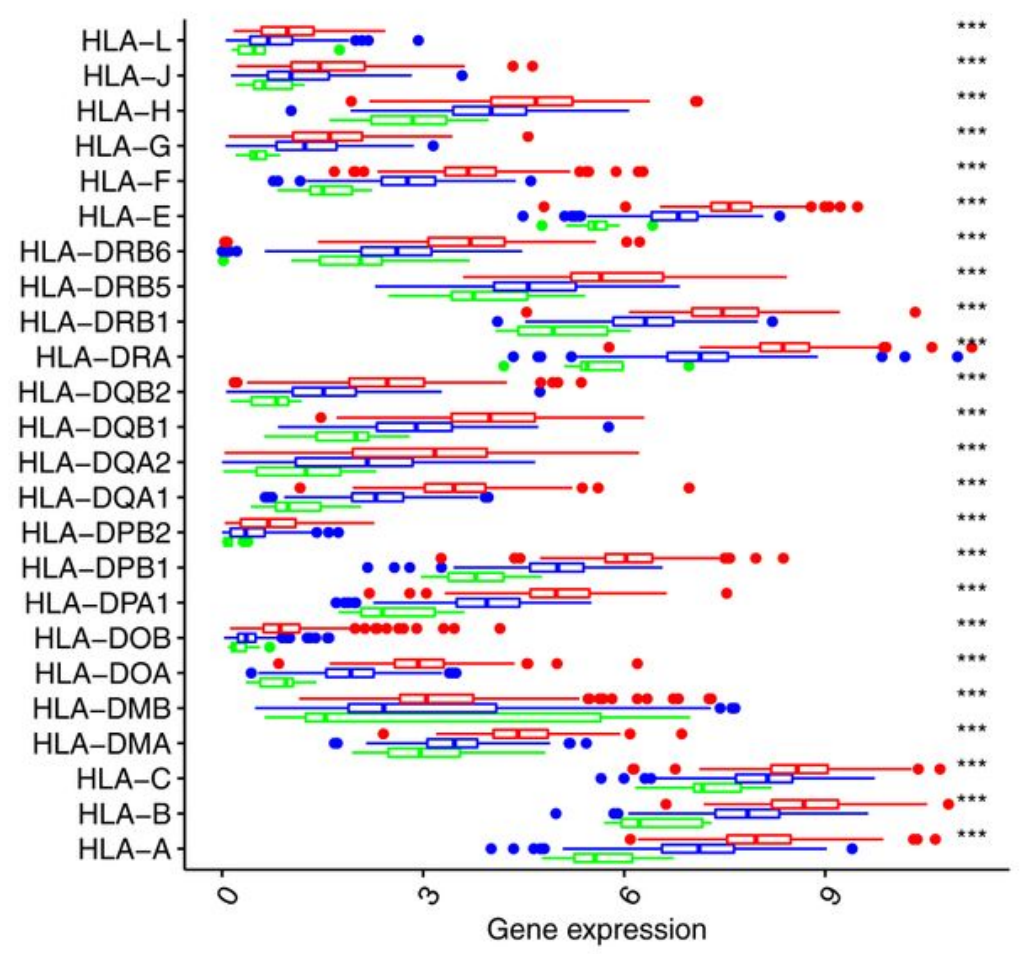

Subtype 自 Immunity_L Immunity_M 官 Immunity_H

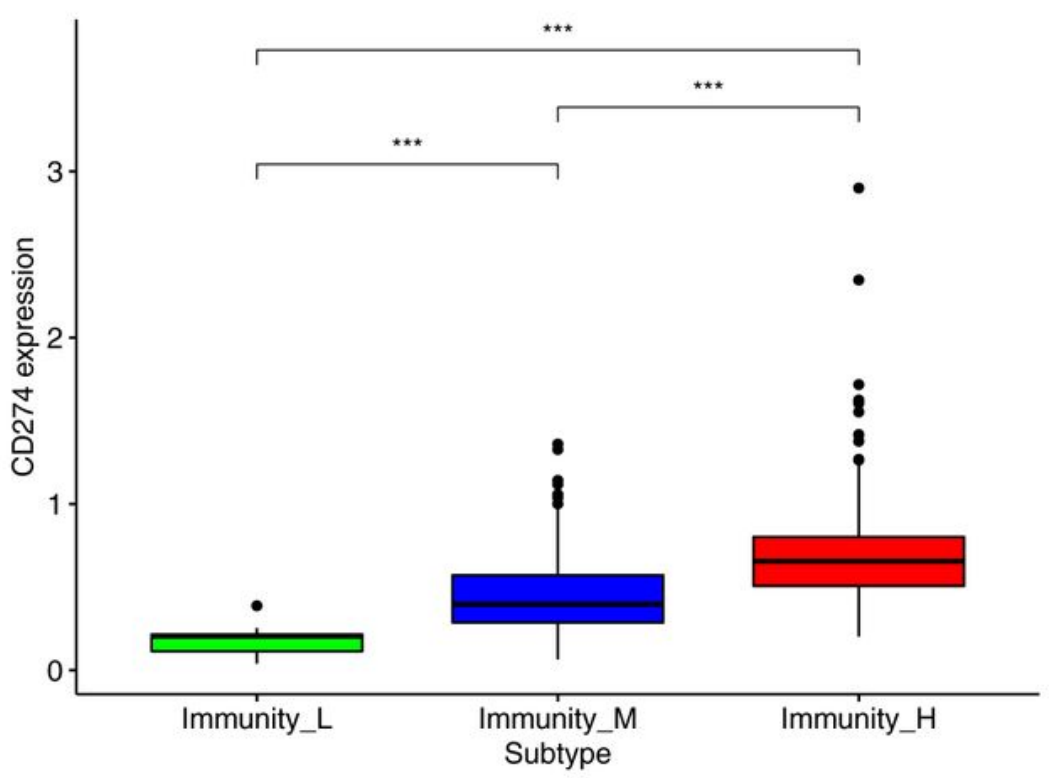

\section{Figure 6}

Besides, the expression of the PD-L1 (CD274) gene increased with the enhancement of immune activity (Figure6a, b). 


\section{Up}

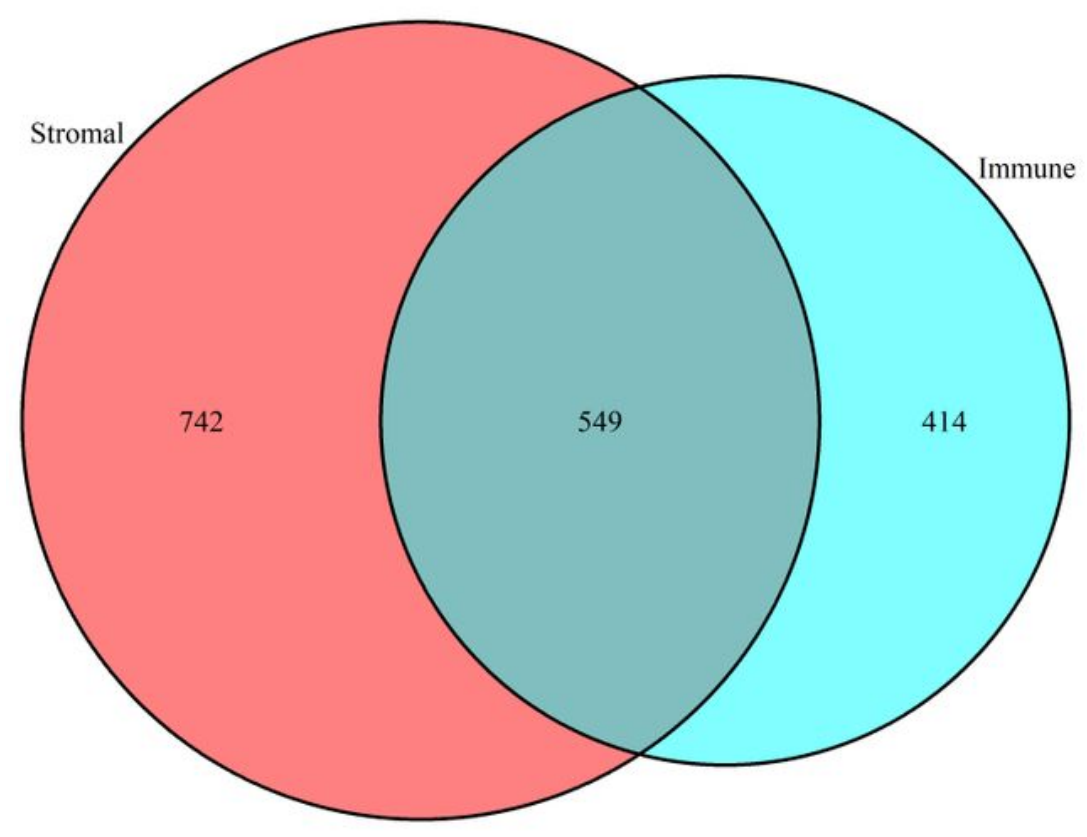

Down

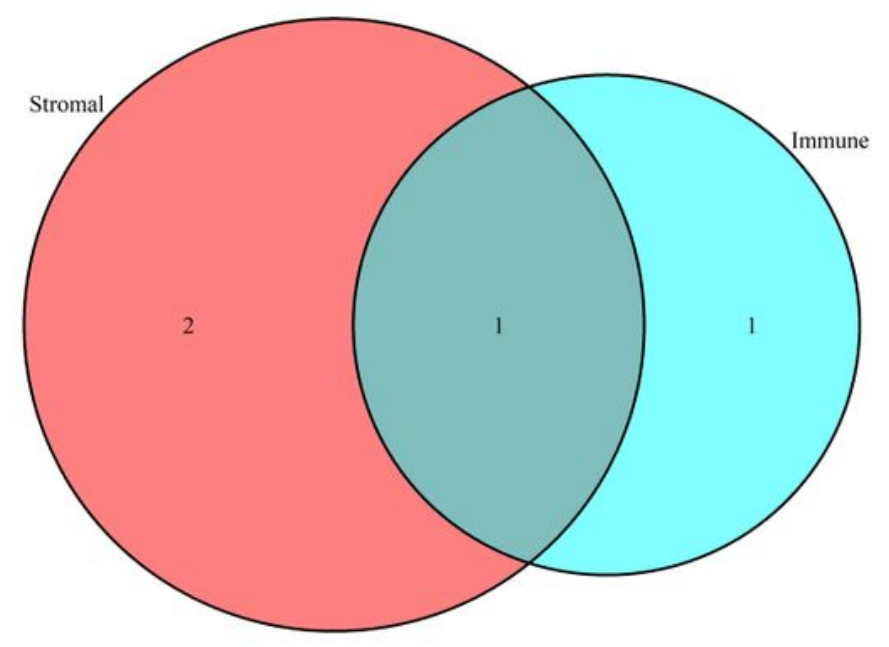

Figure 7

Then 549 up-regulated genes and 1 down-regulated gene were obtained by Venn analysis $\otimes F i g u r e 7 a, b)$. 


\section{Up}

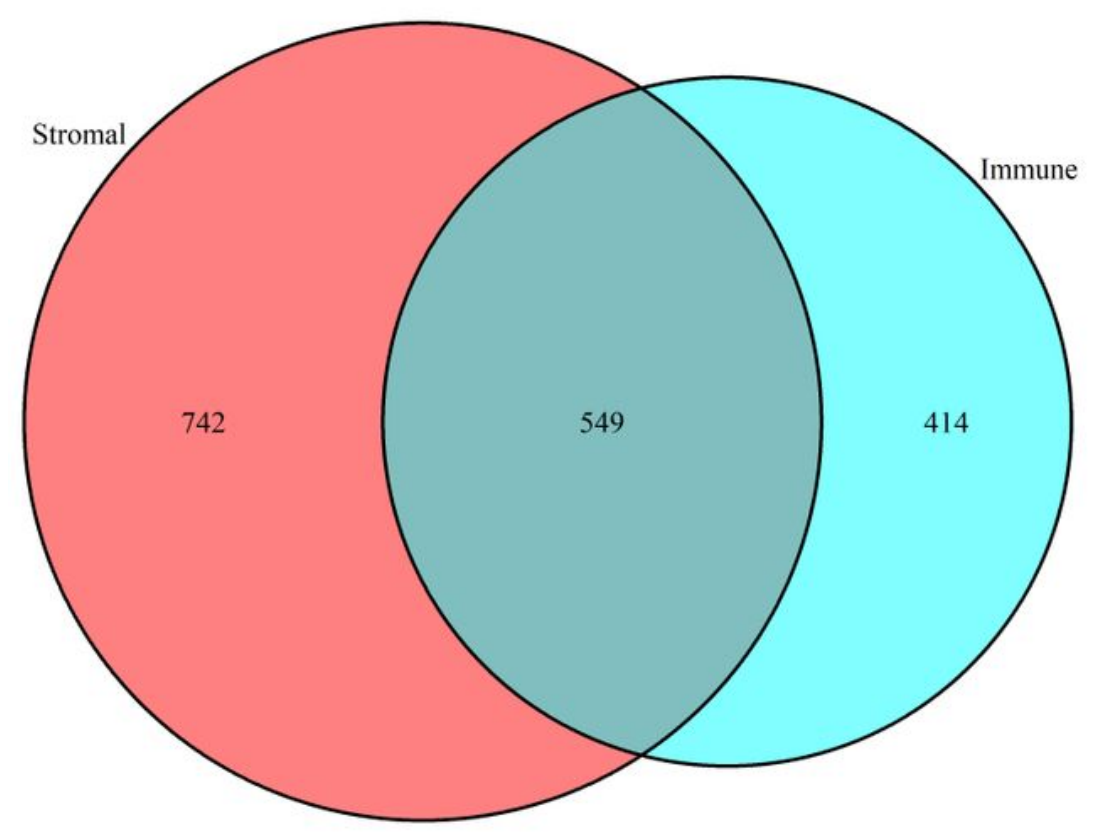

Down

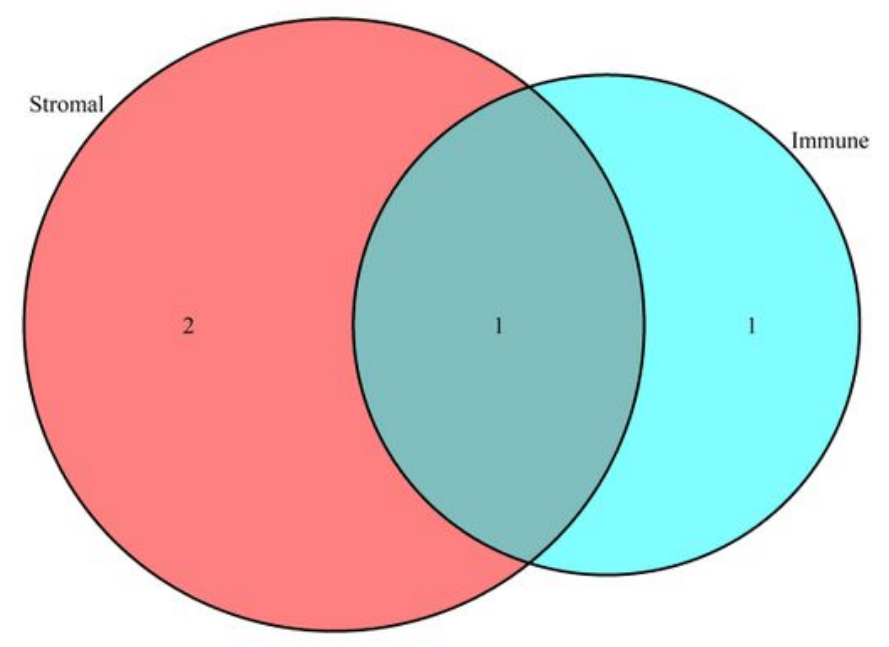

Figure 7

Then 549 up-regulated genes and 1 down-regulated gene were obtained by Venn analysis $\otimes$ Figure7a,b). 

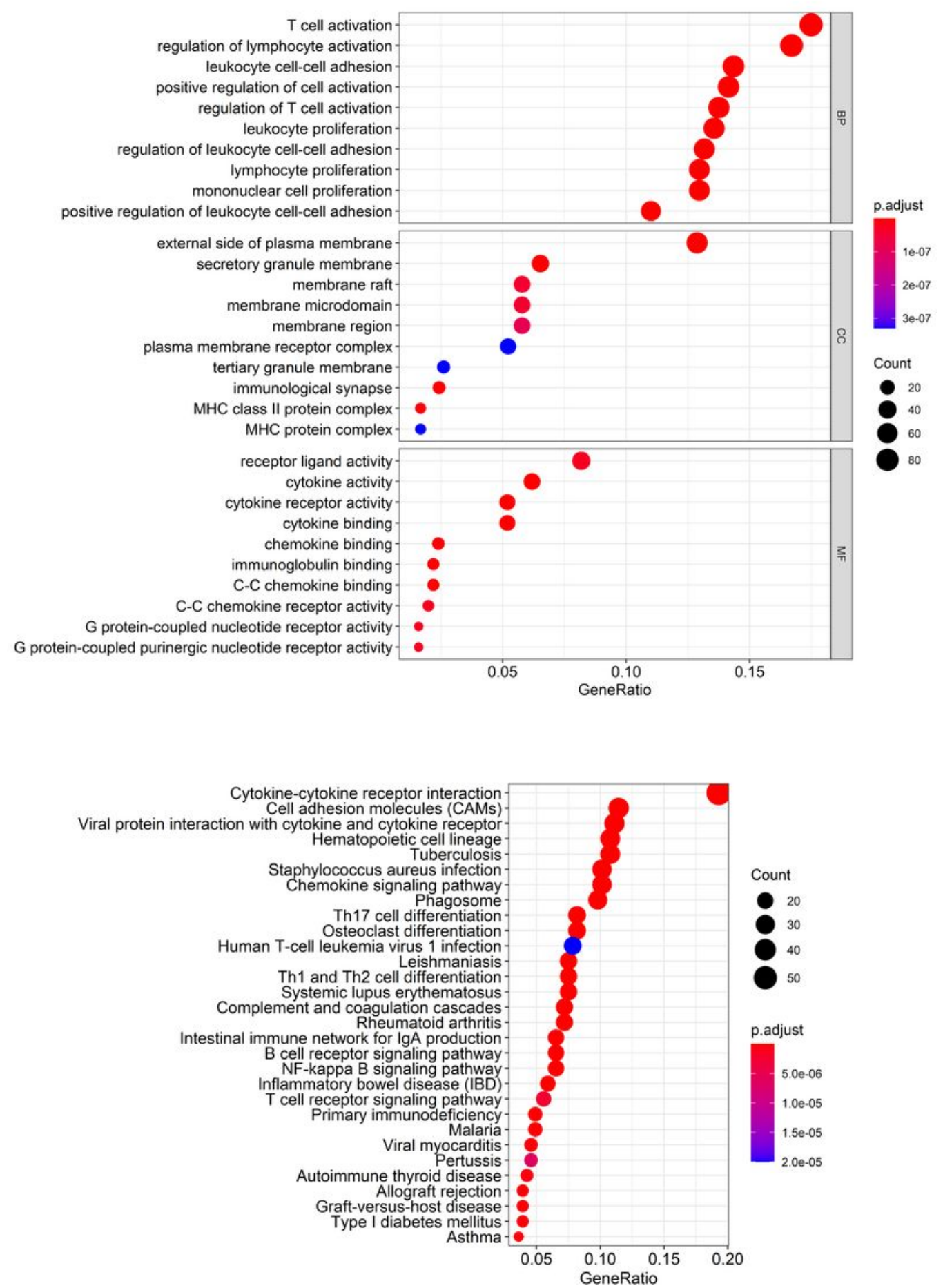

\section{Figure 8}

The main biological pathways involve cytokine-cytokine receptor interaction and cell adhesion molecule CAMS (Figure8a, b). 

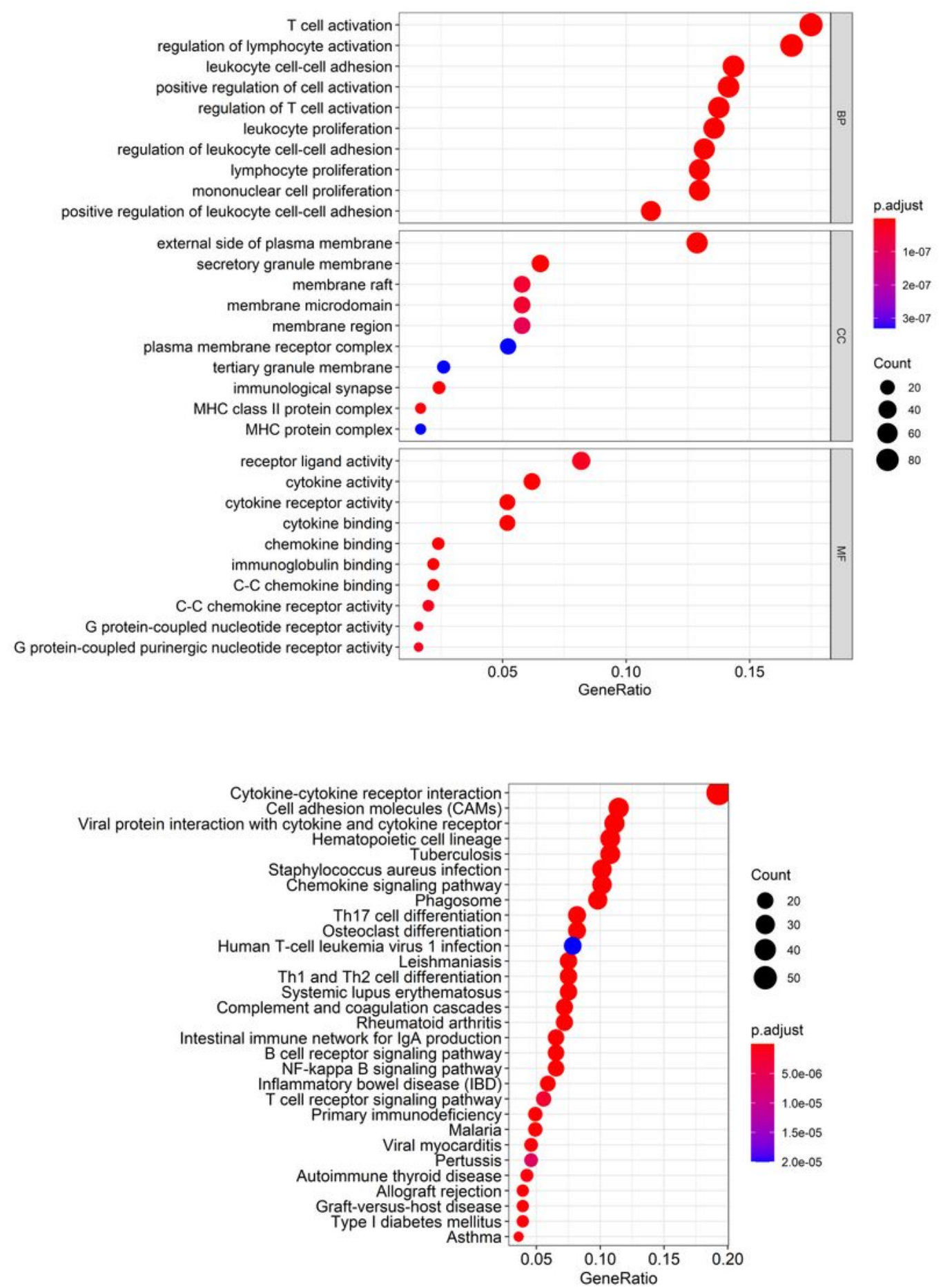

\section{Figure 8}

The main biological pathways involve cytokine-cytokine receptor interaction and cell adhesion molecule CAMS (Figure8a, b). 

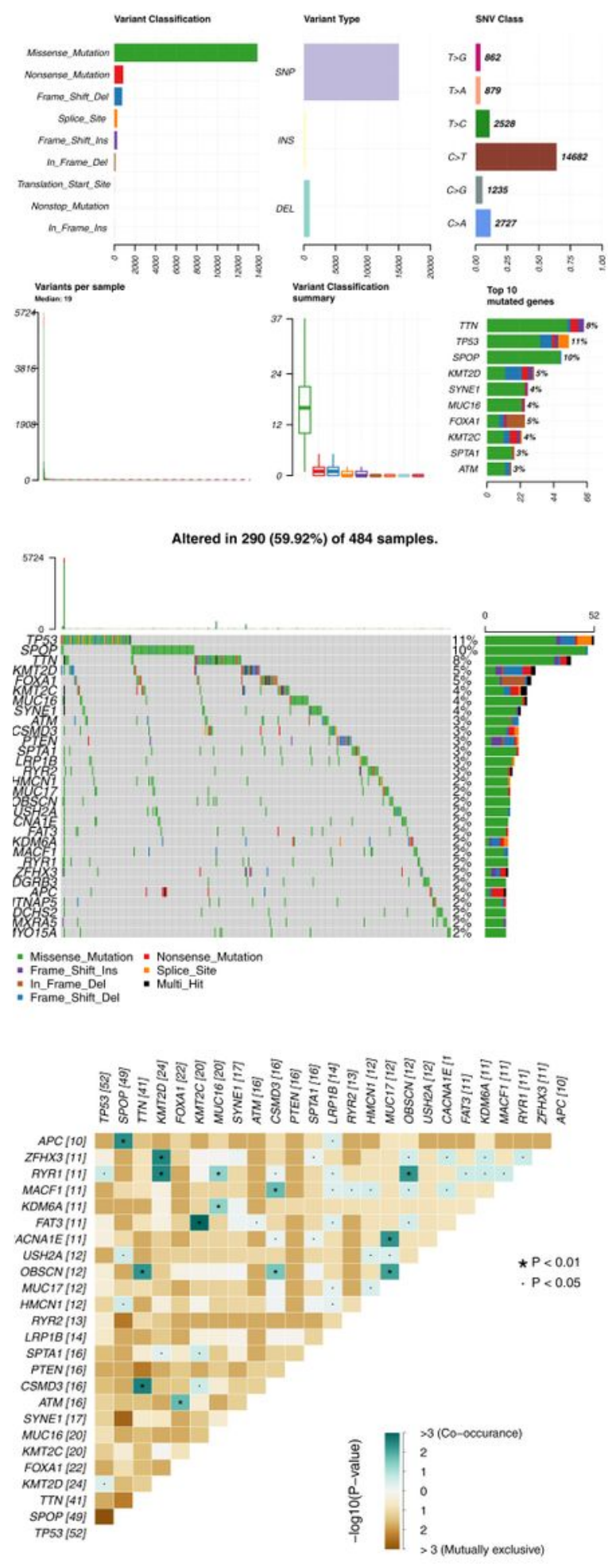

\section{Figure 9}

Among them, KMT2 family genes not only have a high mutation rate but also co-occur with many genes, including FAT3 and KMT2C, ZFHX3, and KMT2D, RYR1, and KMT2D \&Figure9a,b,c). 

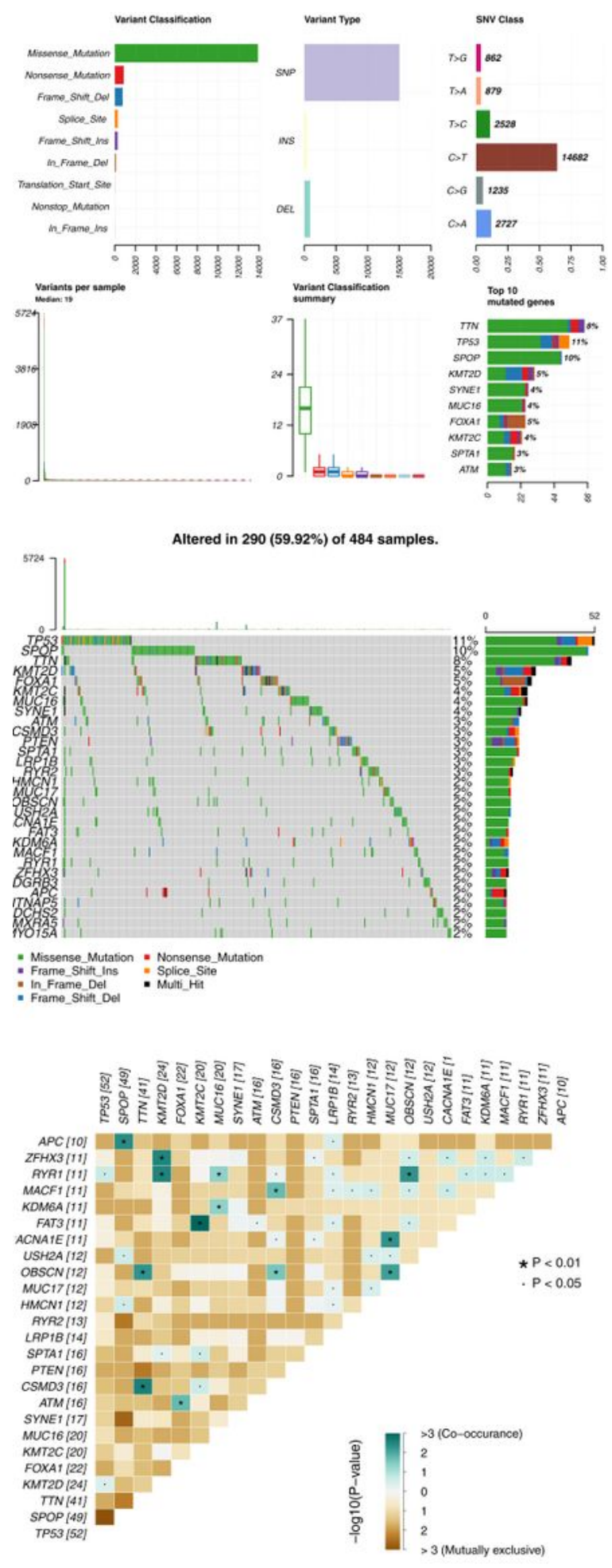

\section{Figure 9}

Among them, KMT2 family genes not only have a high mutation rate but also co-occur with many genes, including FAT3 and KMT2C, ZFHX3, and KMT2D, RYR1, and KMT2D \&Figure9a,b,c). 


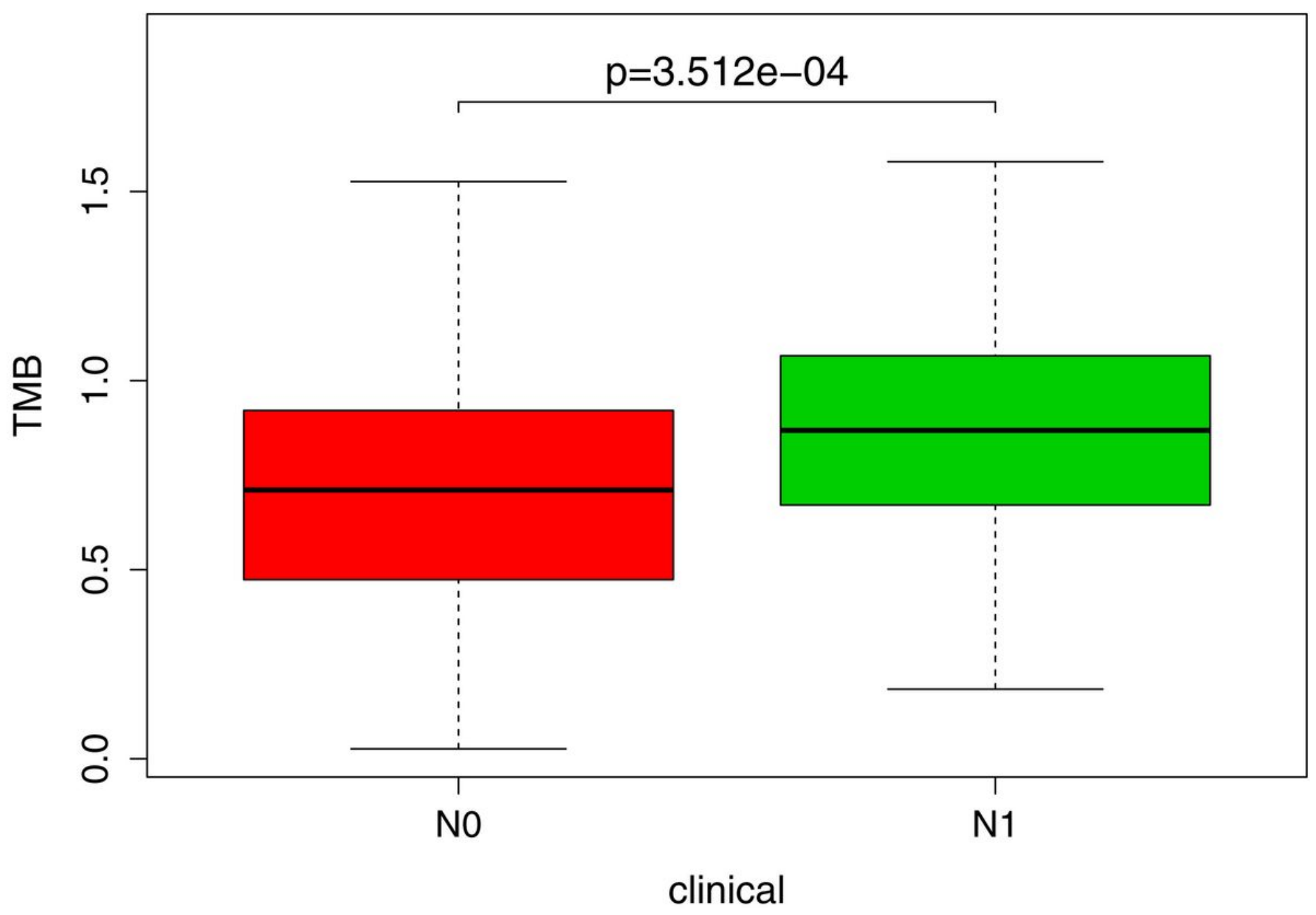

Figure 10

To explore the relationship between TMB value and PCa lymph node metastasis, it was found that the TMB value in the N1 stage was higher than that in the N0 stage in TNM staging $\otimes$ Figure10). 


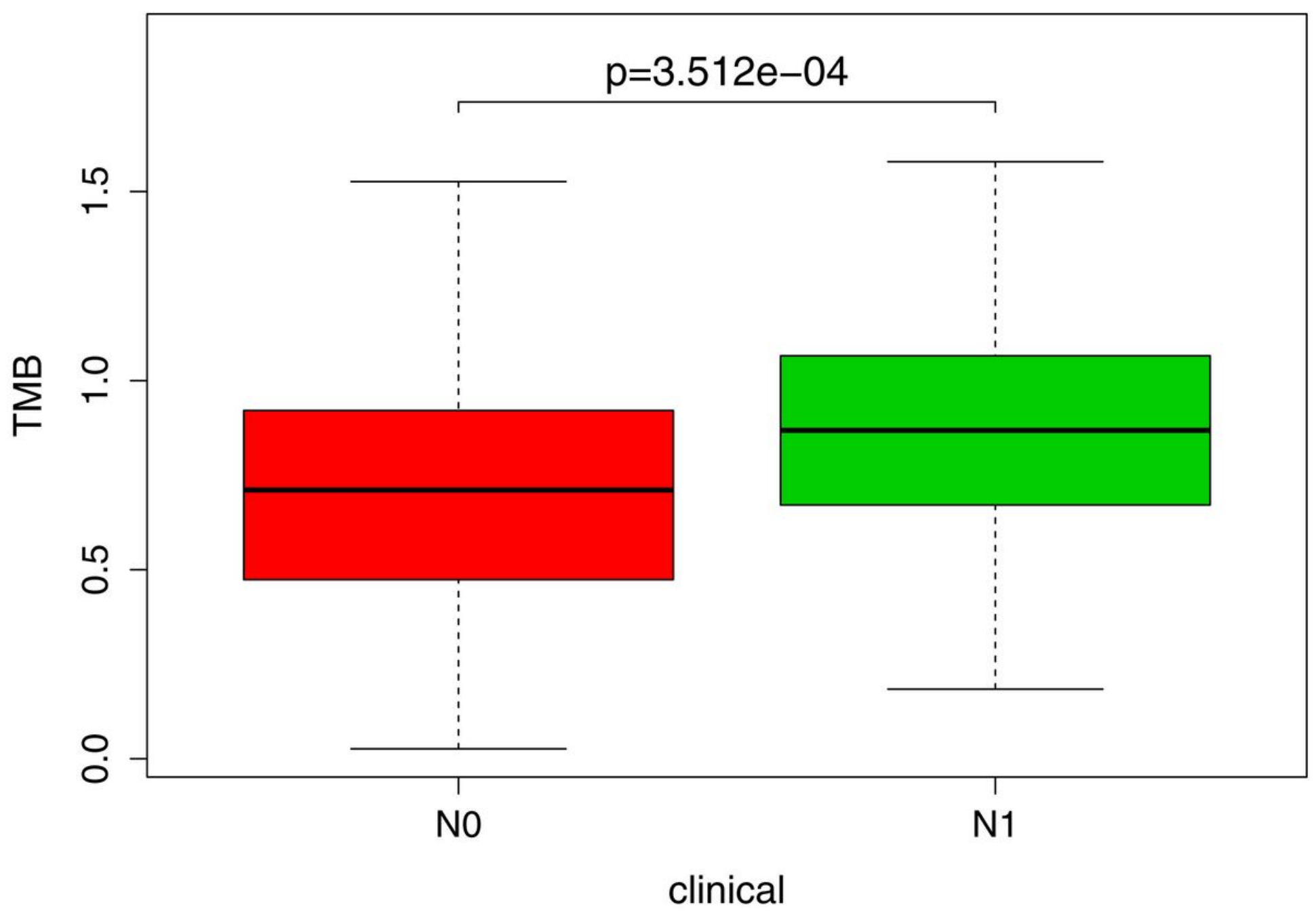

Figure 10

To explore the relationship between TMB value and PCa lymph node metastasis, it was found that the TMB value in the N1 stage was higher than that in the N0 stage in TNM staging $\otimes$ Figure10). 


\section{microenvironment}
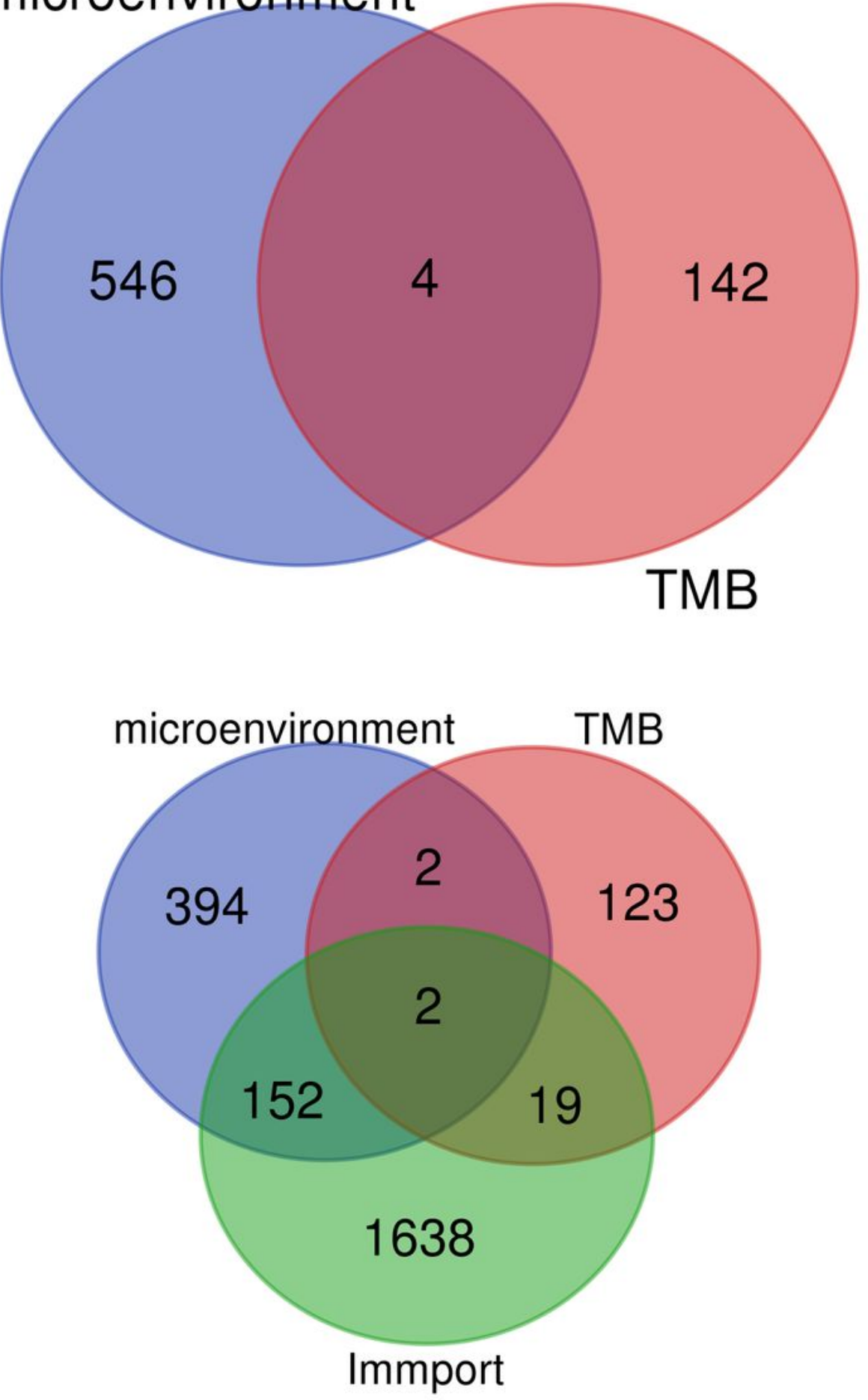

Figure 11

2498 immune-related genes were retrieved from the ImmPort database to screen out the two genes most related to the immune microenvironment, CSF3, and LTF (Figure11a, b). 


\section{microenvironment}
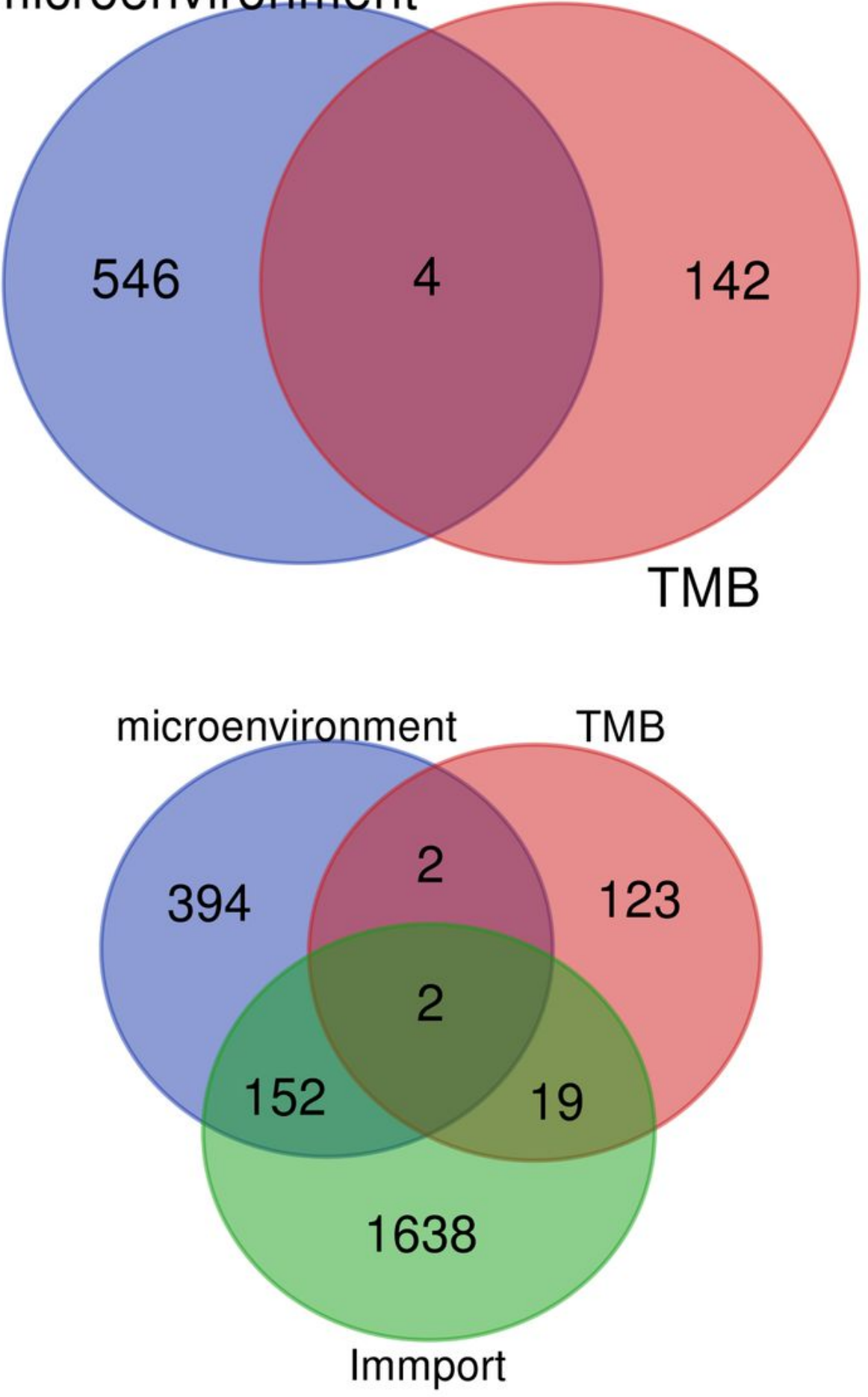

Figure 11

2498 immune-related genes were retrieved from the ImmPort database to screen out the two genes most related to the immune microenvironment, CSF3, and LTF (Figure11a, b). 


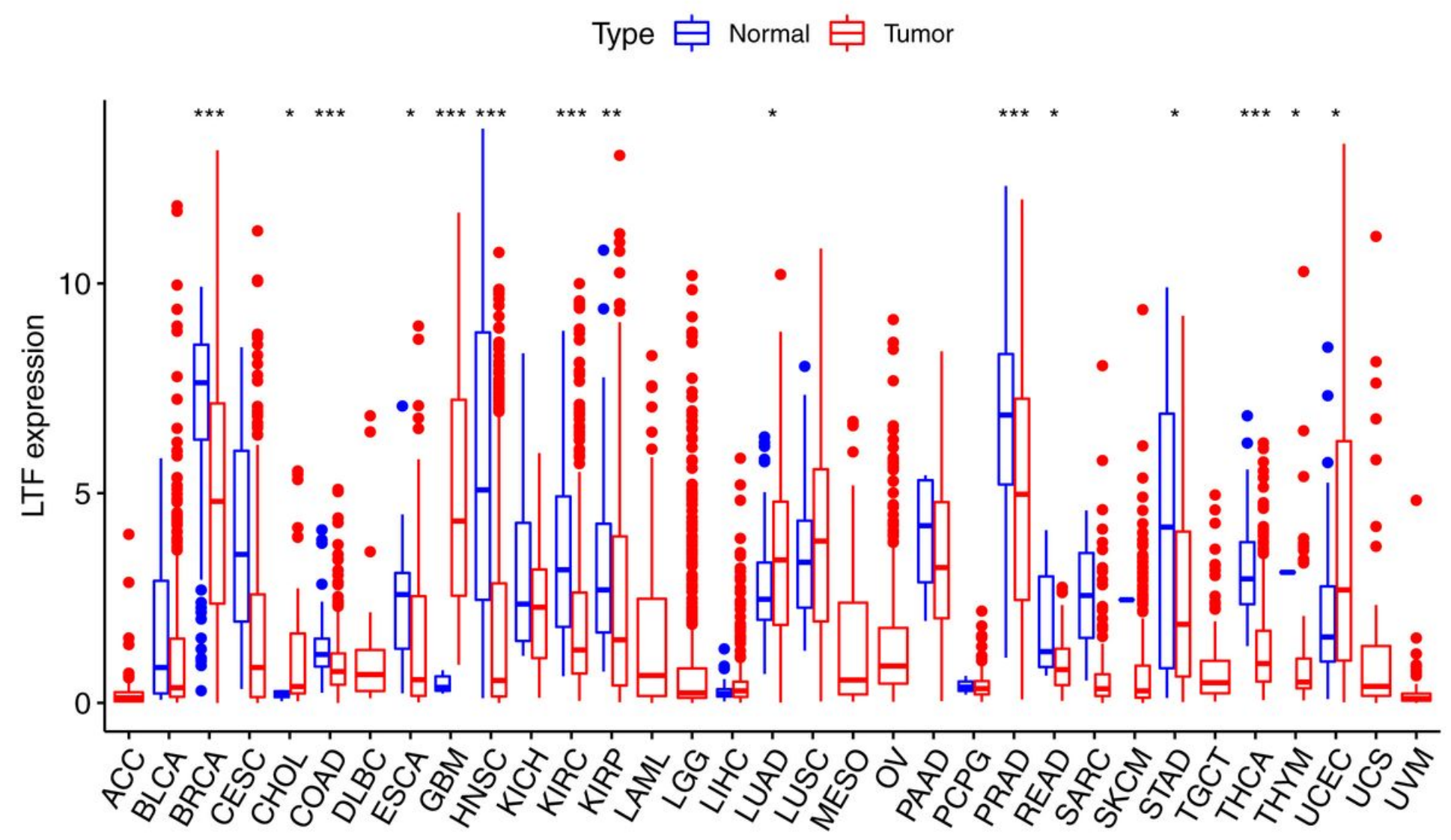

Figure 12

In most of the tumors, the expression level was higher in the normal group than in the tumor group (Figure12). 


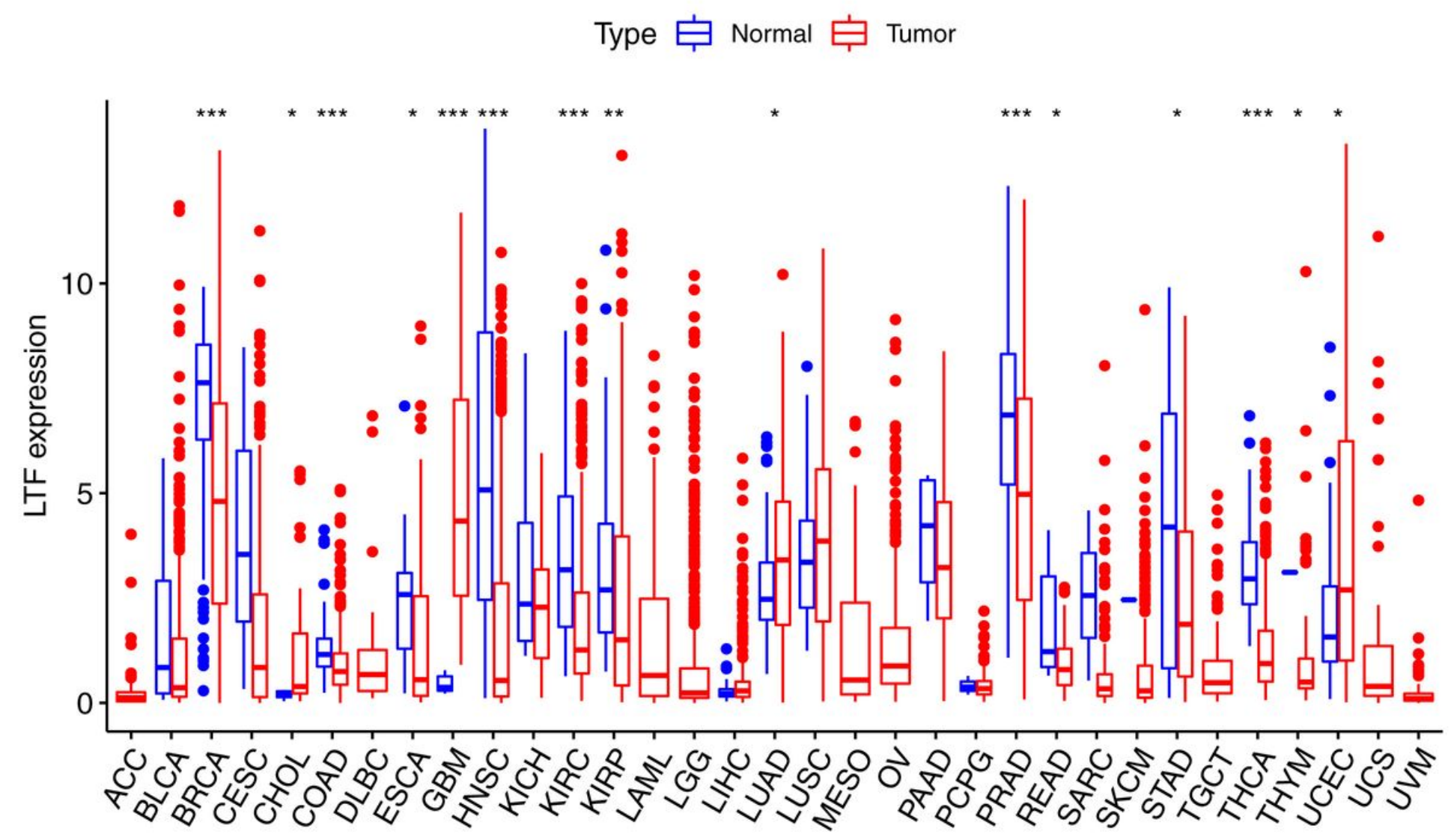

Figure 12

In most of the tumors, the expression level was higher in the normal group than in the tumor group (Figure12). 
Cancer: ACC

LTF levels + high + low
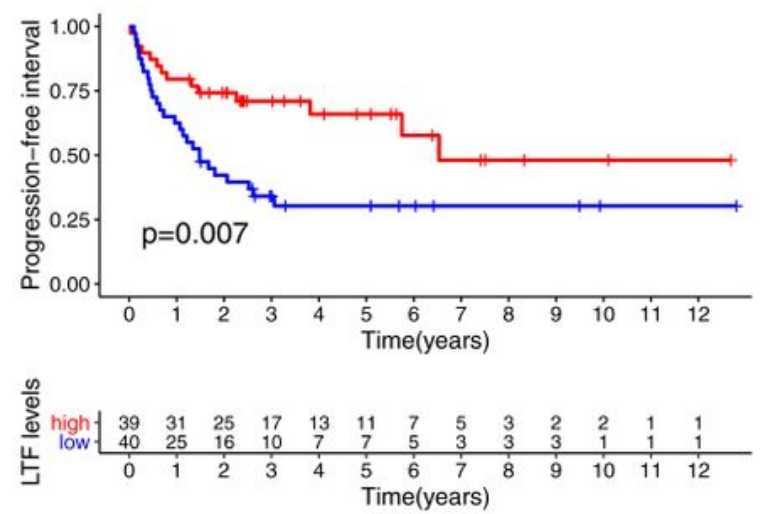

Cancer: PRAD

LTF levels + high + low
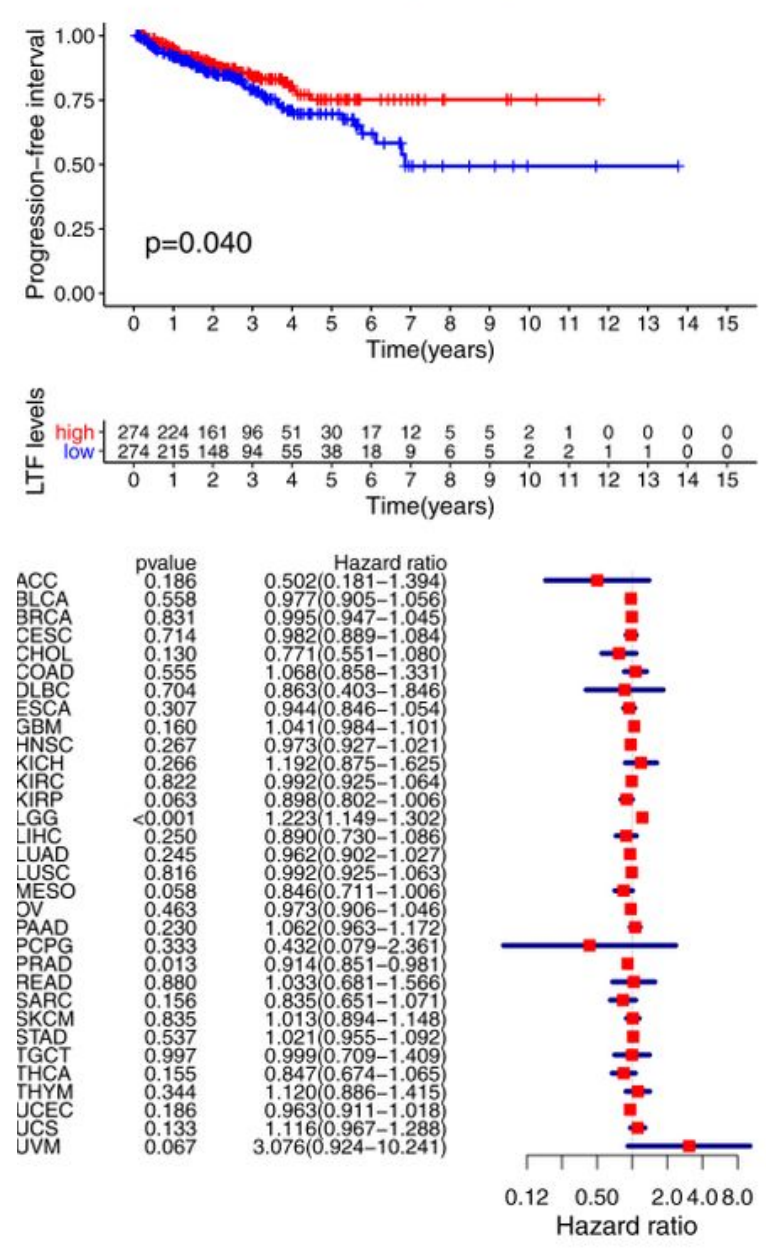

Figure 13

According to the forest plot, there was a correlation between the expression of LTF in PRAD and the survival of PFI, and LTF was a low-risk gene $(H R<1)$ (Figure13a, b, c). 
Cancer: ACC

LTF levels + high + low
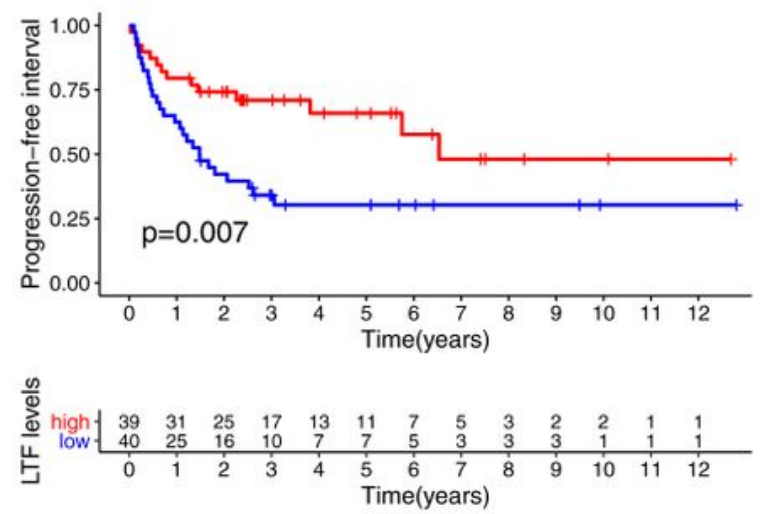

Cancer: PRAD

LTF levels + high + low
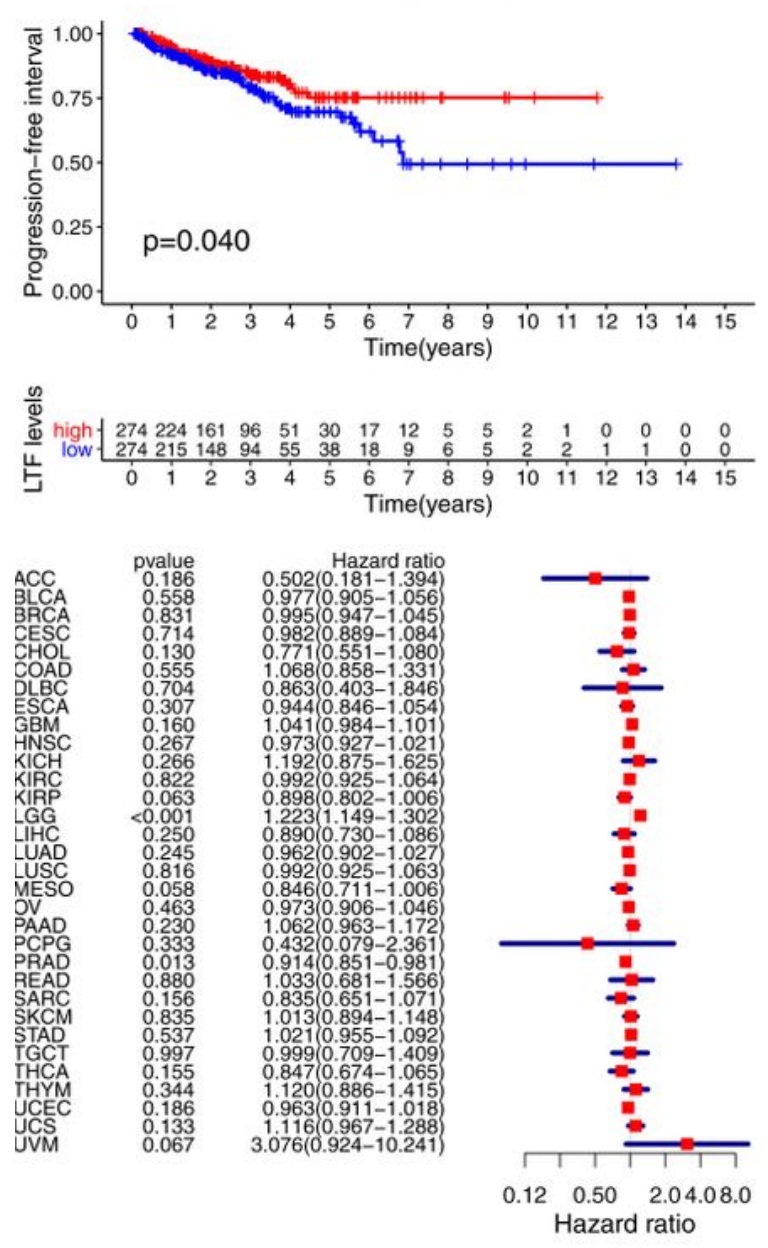

Figure 13

According to the forest plot, there was a correlation between the expression of LTF in PRAD and the survival of PFI, and LTF was a low-risk gene $(H R<1)$ (Figure13a, b, c). 

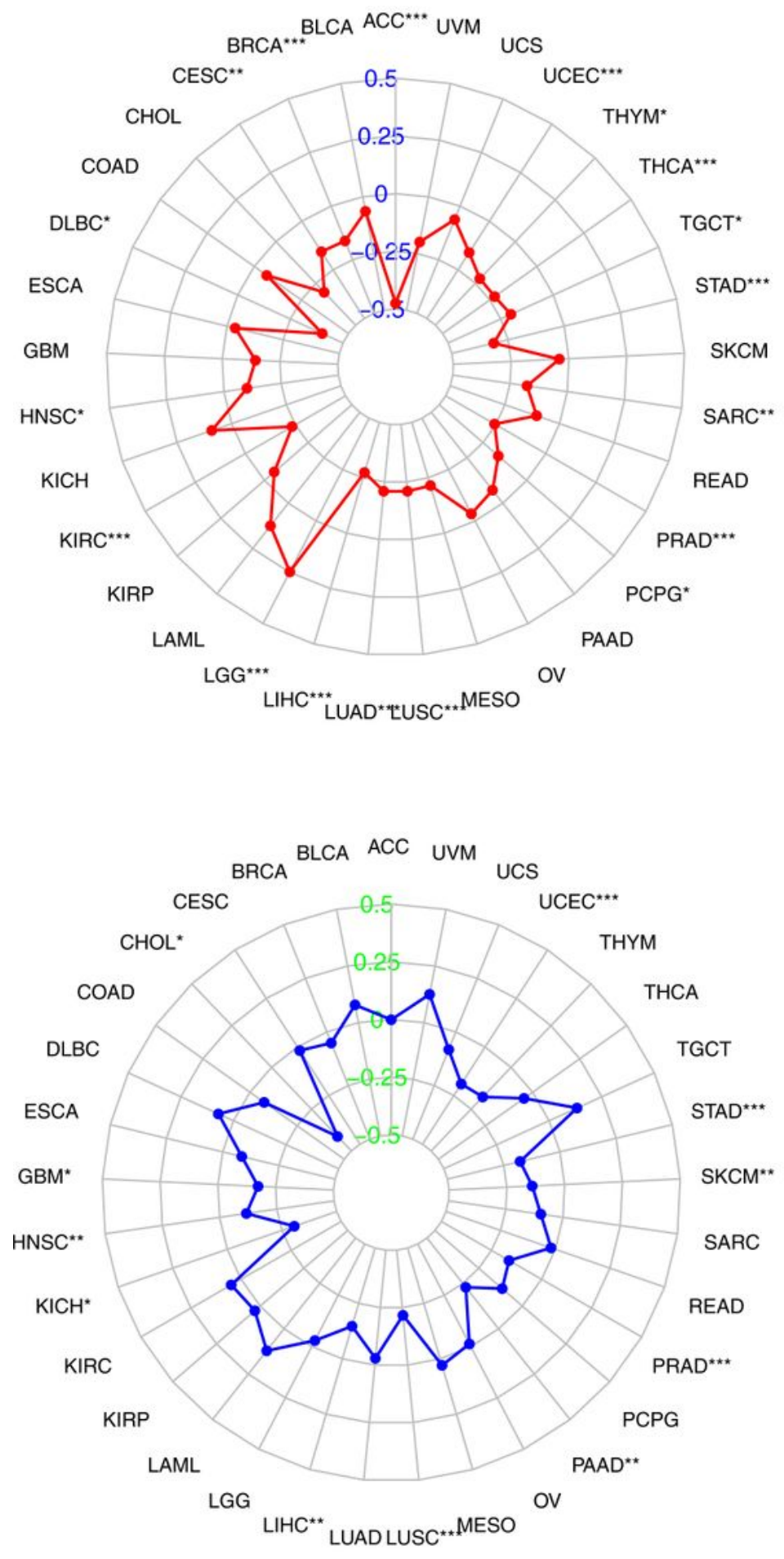

\section{Figure 14}

The expression of LTF in 33 kinds of tumors was correlated with TMB values of 18 kinds of tumors and was negatively correlated except LGG. LTF was negatively correlated with MSI of 11 kinds of tumors including PRAD (Figure14a, b). 

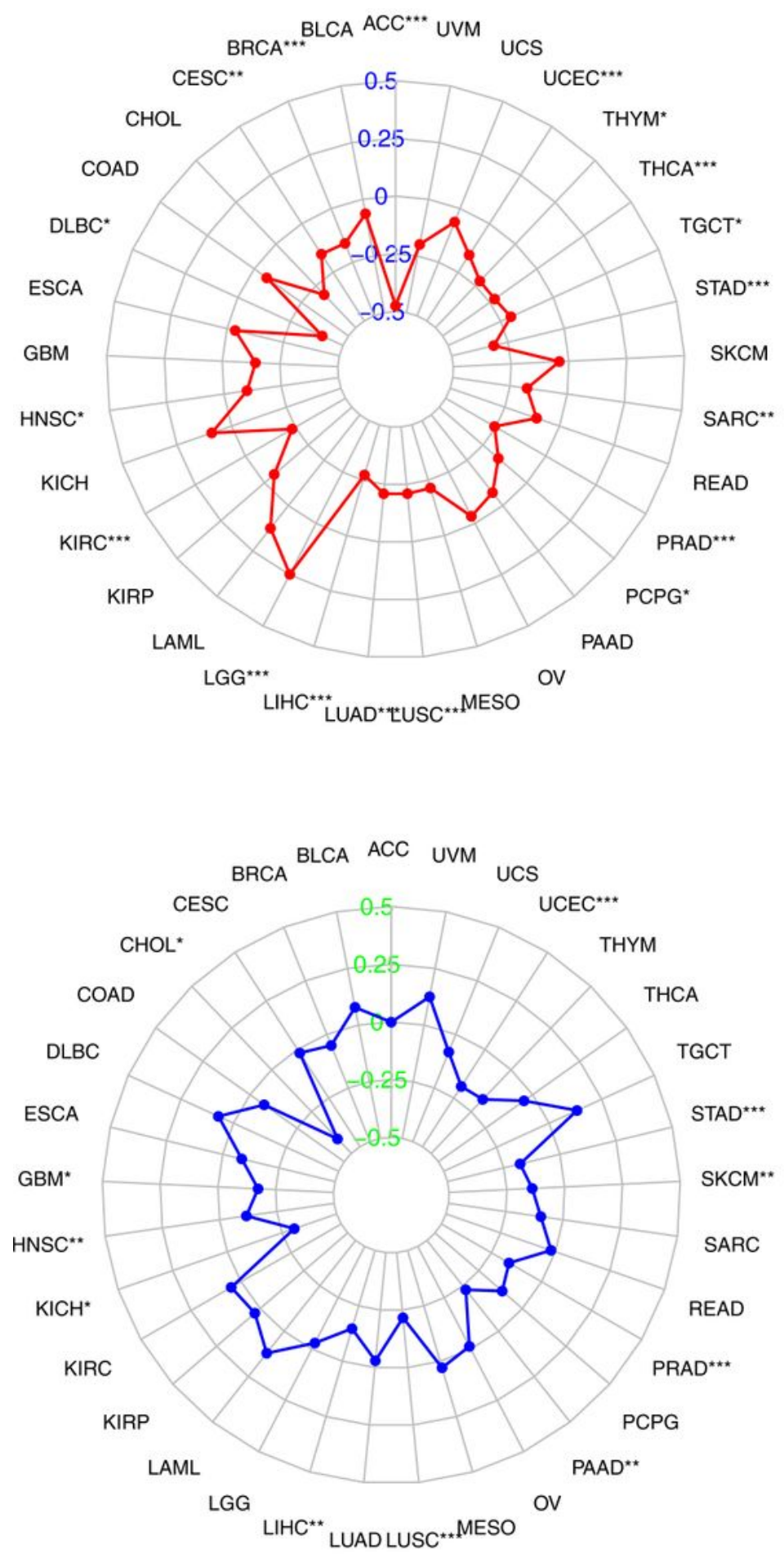

\section{Figure 14}

The expression of LTF in 33 kinds of tumors was correlated with TMB values of 18 kinds of tumors and was negatively correlated except LGG. LTF was negatively correlated with MSI of 11 kinds of tumors including PRAD (Figure14a, b). 
A
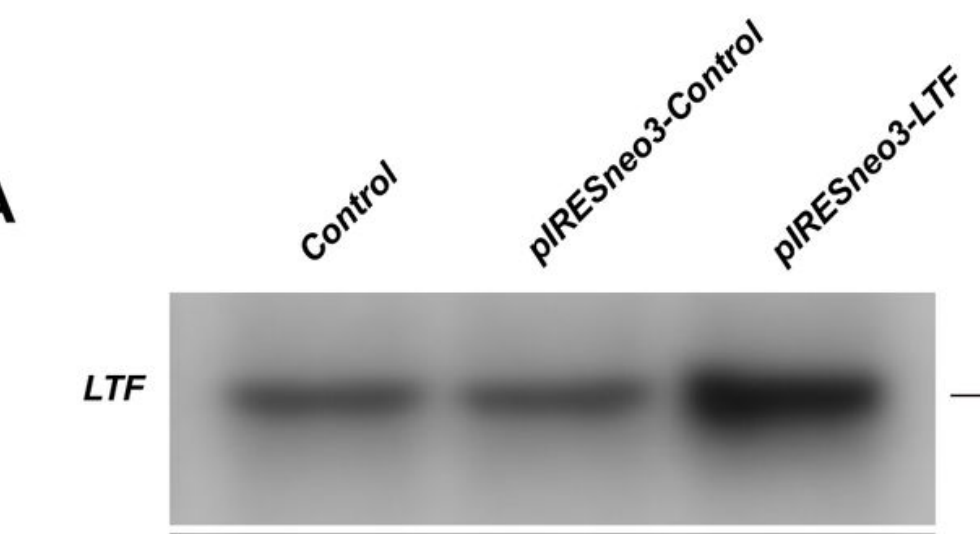

GAPDH

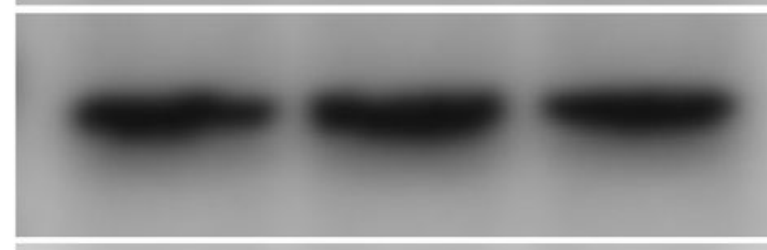

STAT3

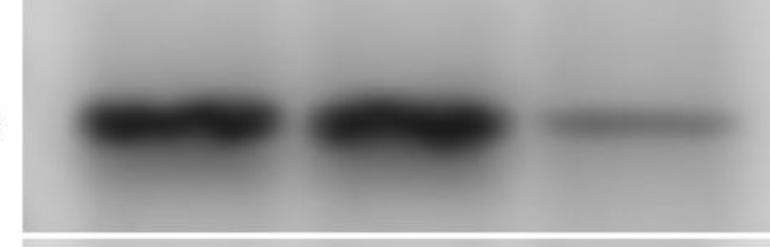

GAPDH

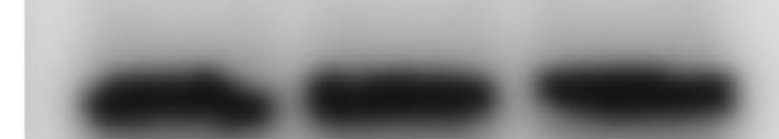

$-88$

$-36$

GM-CSF

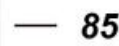

$-36$

C

B
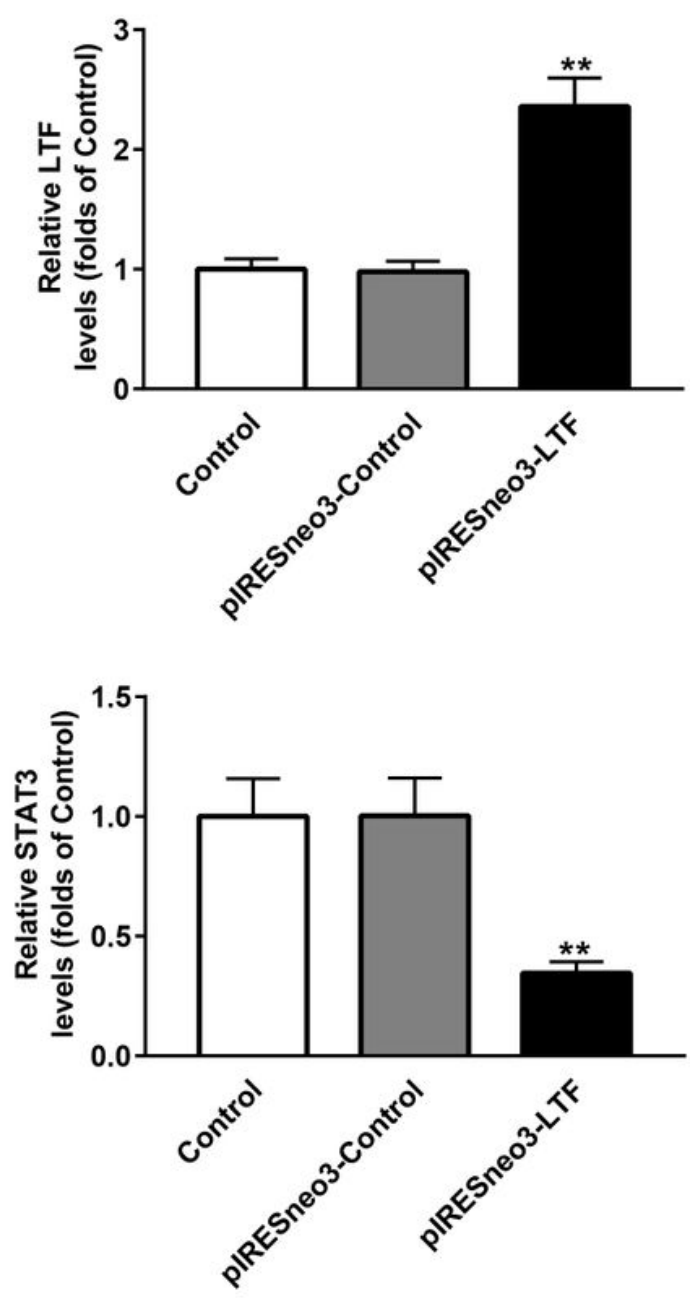

D

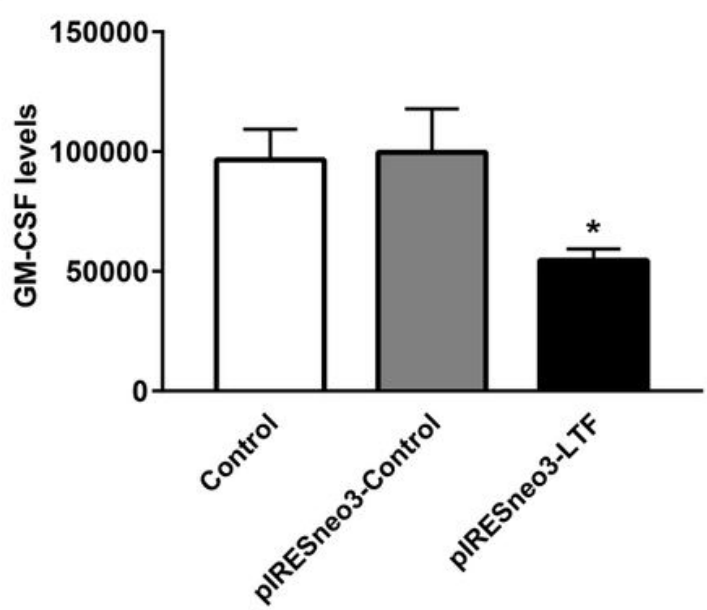

Figure 15

The results showed that compared with the pIRESneo3-Control group, the content of LTF protein in the pIRESneo3-LTF group increased significantly, while the content of STAT3 and GM-CSF protein decreased significantly (Figure15). 
A
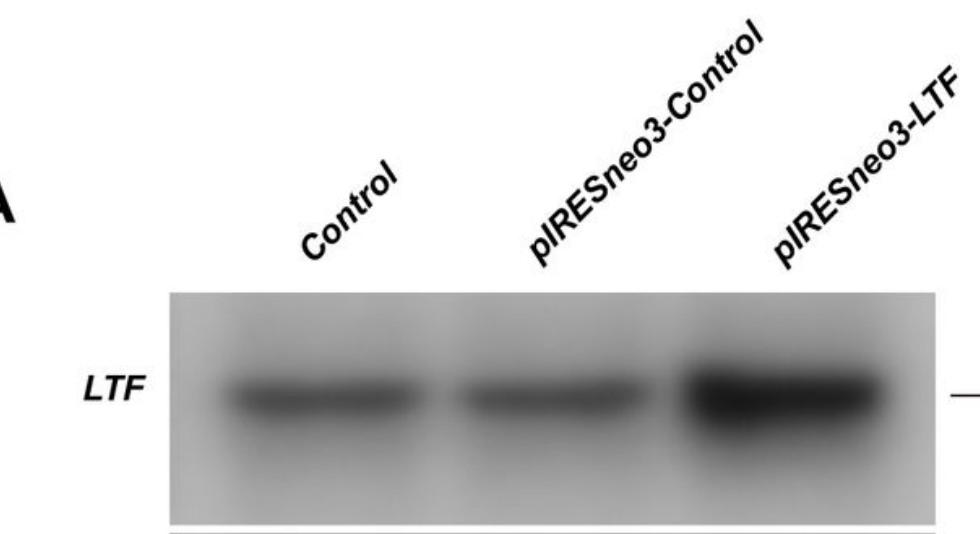

GAPDH

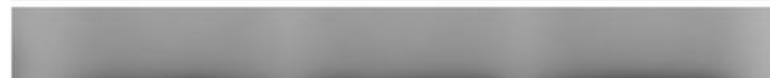

STAT3

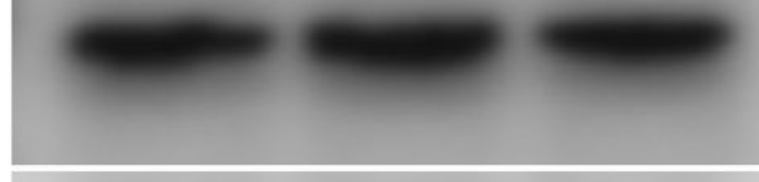

STAT3

GAPDH

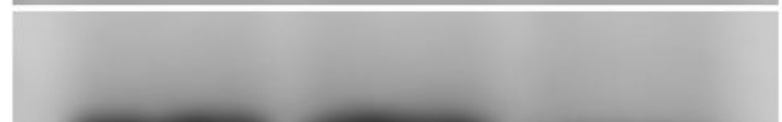

$-85$

$-88$

GM-CSF

$-36$

36

C

B
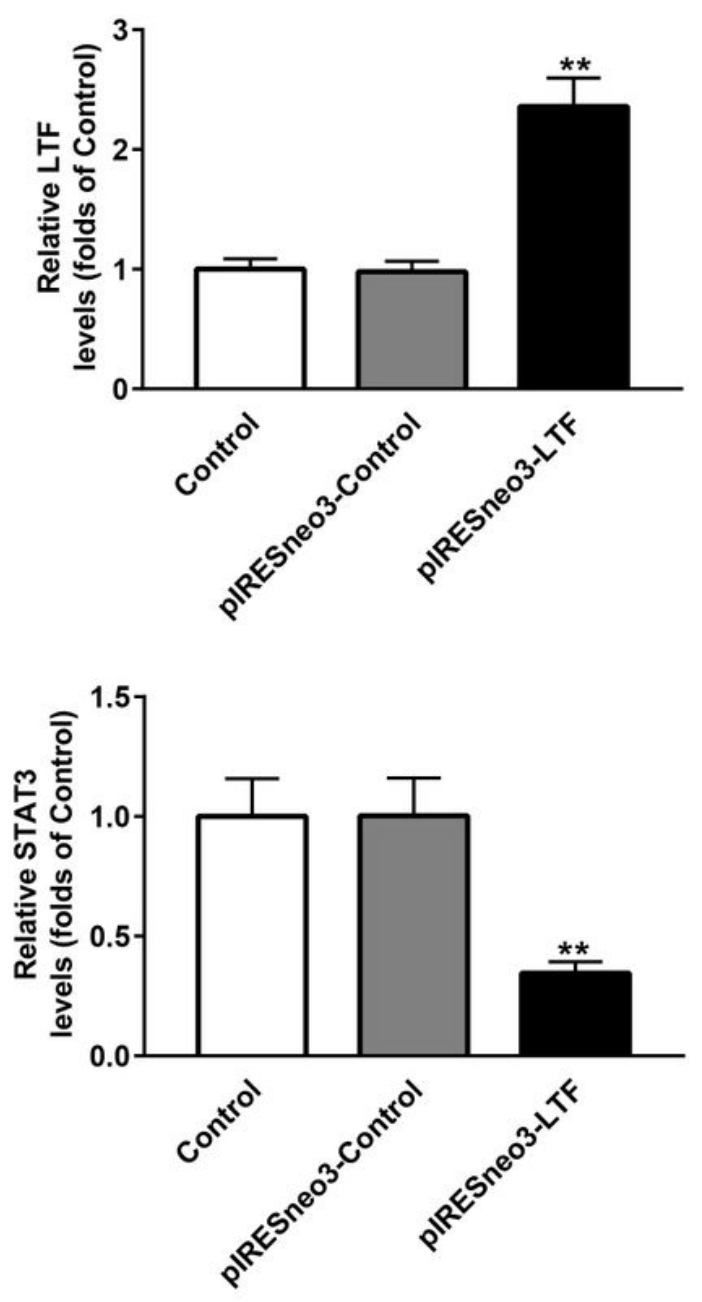

D

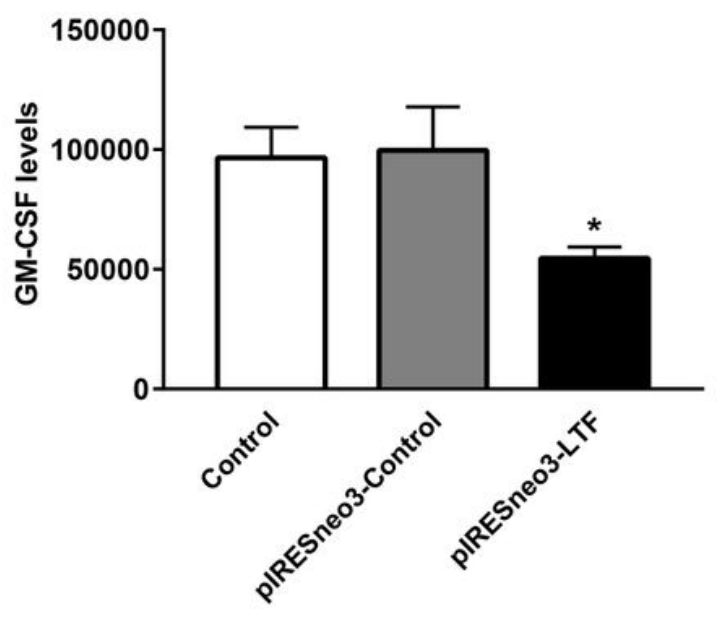

Figure 15

The results showed that compared with the pIRESneo3-Control group, the content of LTF protein in the pIRESneo3-LTF group increased significantly, while the content of STAT3 and GM-CSF protein decreased significantly (Figure15).

\section{Supplementary Files}


This is a list of supplementary files associated with this preprint. Click to download.

- Westernblotexperiment.rar

- Westernblotexperiment.rar

- Table1.xlsx

- Table1.xlsx

- Table2.xlsx

- Table2.xlsx

- Table3.xlsx

- Table3.xlsx

- Table4.xlsx

- Table4.xlsx

- Table5a.xlsx

- Table5a.xIsx

- Table5b.xIsx

- Table5b.xIsx

- Table6.xIsx

- Table6.xlsx

- Table7.xlsx

- Table7.xlsx

- Table8.xlsx

- Table8.xlsx 